\title{
Neuro-Fuzzy modal control of smart laminated composite structures modeled via mixed theory and high-order shear deformation theory
}

\author{
Joana Pereira Repinaldo ${ }^{a}$ (D) , Albert Willian Faria ${ }^{b}$ (D), Rodrigo Alves e Silva ${ }^{c}$ (D), Edson Hideki Koroishia* (D), \\ Fabian Andres Lara-Molina ${ }^{a}$ (i)
}

a Departamento de Engenharia Mecânica, Universidade Tecnológica Federal do Paraná (UTFPR), Cornélio Procópio-PR, Brasil.

E-mail: joanarepinaldo@hotmail.com, edsonh@utfpr.edu.br, fabianmolina@utfpr.edu.br

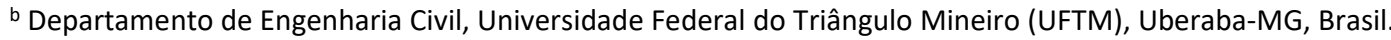

E-mail: albert.faria@uftm.edu.br

c GeoEngineering Centre at Queens-RMC, Queens University, Kingston, ON, Canada. E-mail: r.esilva@queensu.ca

* Corresponding author

http://dx.doi.org/10.1590/1679-78255873

\begin{abstract}
The present study develops and applies a neuro-fuzzy modal vibration control of smart laminated composite structures with piezoelectric layers via Mixed theory. Differently from previous studies, the composite structures in this paper are modeled via the Mixed Theory using the High-order Shear Deformation Theory (HSDT) theory. The Mixed Theory adopts a single layer when representing the mechanical displacement field, through HSDT theory, and multiple layers (Layerwise theory) for the electrical degrees of freedom. The Mixed Theory is computationally implemented in the Matlab ${ }^{\circledR}$ software using a plate-type element called Serendipity. Moreover, a neuro-fuzzy active vibration controller is implemented to attenuate the vibration of the smart composite structures. The numerical results validate the electric-mechanical coupling adopted, showing the importance of the mixed theory in the static and dynamic modeling of slender beams and plates with piezoelectric layers. Finally, the results of the robustness analysis indicate that the neuro-fuzzy controller has benefits compared with the linear quadratic regulator.
\end{abstract}

\section{Keywords}

laminated composite materials, smart structures, active vibration control, active modal control, finite element, high-order shear deformation theory, linear quadratic regulator, linear matrix inequalities, neuro-fuzzy controller

\section{Graphical Abstract}
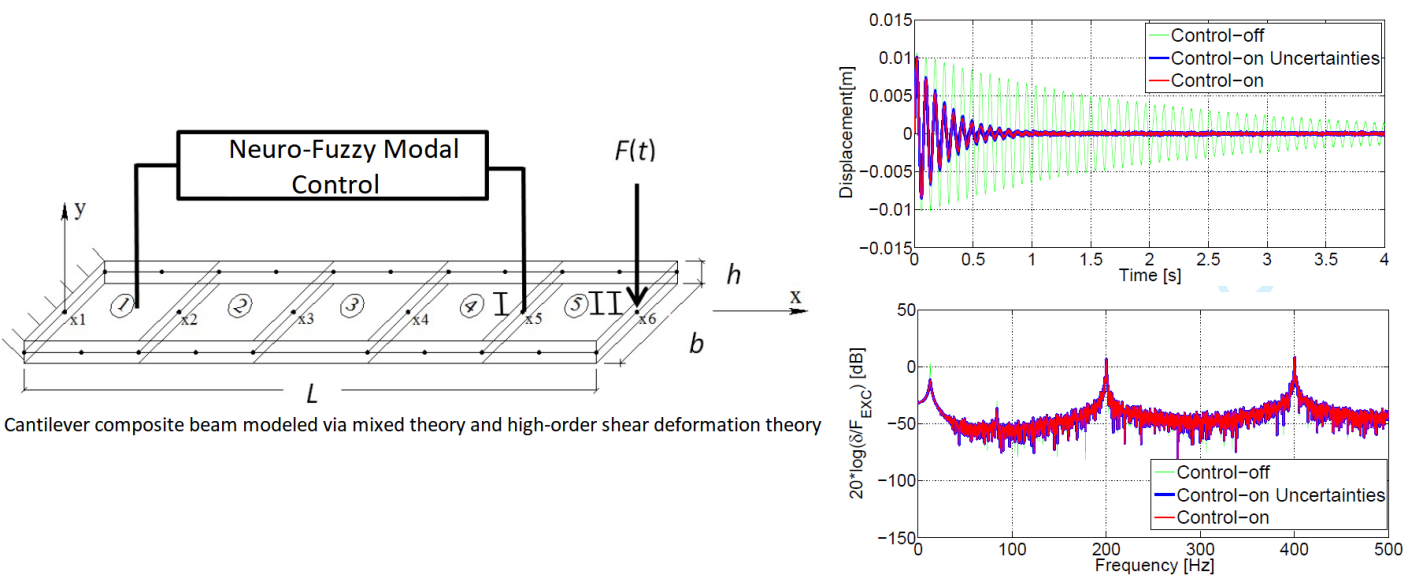

Received: November 21, 2019. In Revised Form: February 19, 2020. Accepted: February 20, 2020. Available online: February 20, 2020 https://doi.org/10.1590/1679-78255873

(i) Latin American Journal of Solids and Structures. ISSN 1679-7825. Copyright (c) 2020. This is an Open Access article distributed under the terms of the Creative Commons Attribution License, which permits unrestricted use, distribution, and reproduction in any medium, provided the original work is properly cited. 


\section{INTRODUCTION}

Laminated composite materials have been applied to numerous manufactured products due to their high resistance/weight relation. Additionally, their stratified structure with overlapping layers and different fiber orientations enables an optimized design in many specific operating conditions, either in elastic or dynamic regimen Reddy (2003).

In the current state of technological development, laminated composite materials are integrated with several state-of-the-art types of equipment, notably within the aerospace sector. In this context, an important aspect to be considered is the necessary instrumentation for the acquisition of static or dynamic responses. This acquisition process must be executed with the aid of less intrusive sensors, and actuators of high reliability. Piezoelectric materials may offer interesting alternatives within such aspects, since they may be integrated with the structure either by being attached to its surface or embedded inside (Elahi et al. (2018) and Memmolo et al. (2019)). The combination of sensors, actuators, structures, and controlling mechanisms comprise the so-called smart structures.

Amongst different existing techniques for modeling smart composite structures, the Finite Element Method (FEM) is one of the most adequate approaches. The finite element method presents advantageous characteristics of modeling flexibility and relative ease for numerical implementations.

The scientific literature has brought a wide variety of theories dealing with finite element formulations of smart composite structures, each of which presenting its own favorable and unfavorable features of accuracy, application domain and computational effort (Reddy, 2003; Chee, 2000).

The Third-order Shear Deformation Theory, also known as Higher-order Shear Deformation Theory (HSDT), approximates the mechanical variables using a third-order polynomial function. The HSDT approach provides better approximations of numerical values obtained through 3D Elasticity Theory than the First-order Shear Deformation Theory (FSDT), another tool applied to the modelling of composite structures. Both FSDT and HSDT are classified as Equivalent Single Layer theories within the literature. Apart from this classification, there is also the Discrete Equivalent Layer theories (Layerwise), in which the displacements are continuous functions along the coordinate of the laminate thickness. According to Reddy (2003), whenever the focus is aimed at studying local effects in composite materials such as delamination mechanisms between adjacent layers, fiber rupture and edge effects, Layerwise theories are more appropriate than those formulated with the Equivalent Single Layer approach, which is rather recommended for analyses involving global performances of the composite laminate (e.g. the determination of natural frequencies, vibration modes and displacements). This is why HSDT has been adopted herein.

The Mixed Theory incorporates the mechanical behavior of single layers together with the electrical behavior of multiple layers (Layerwise). In this theory, the mechanical displacement field may be approximated by either the First (FSDT) or High-order Shear Deformation Theory (HSDT), while the electrical variables are approximated by the Layewise Theory. Both theories are integrated into the finite element formulation through Hamilton's Variational Principle (HVP), generating groups of equations that can be solved using appropriate boundary conditions.

Excessive vibrations within structures are undesirable if one wants to achieve mechanical systems with optimized performance. These vibrations can be hampered through vibration control techniques, which might include passive, active and semi-active control systems. Passive control in smart structures can be made with the use of piezoelectric and viscoelastic materials (Manoharan et al., 2015), thus its performance is sensitive to the variations of the system parameters.

Active control approaches employed to the control of vibrations in smart structures are broadly described in the scientific literature. This technique utilizes secondary forces applied to the structure by a controller to minimize its vibrations (Zhang et al., 2015, Rahmani and Shenas,2017). The semi-active techniques comprise the combination of the previous two (Wang et al., 2012). There has been an increasing number of works dedicated to the active control of vibrations in smart structures based upon the linear quadratic regulator (LQR) (Schulz et al., 2013; Koroishi et al., 2015, Mirghaffari and Rahmani, 2017; Xu et al., 2017). The LQR optimal control design is aimed at minimizing an objective function or a performance index.

The smart control, based on neural networks and Fuzzy logic, has emerged as an alternative to the strategies dependent on the system model since it does not require an accurate mathematical formulation. The performance of the Fuzzy controller depends on the effective determination of the control parameters and linguistic rules, but the incorporation of neural networks facilitates the optimization of such parameters. The Neuro-Fuzzy systems join the knowledge of the fuzzy logic specialist with the learning and optimization capacity of the neural networks. Some works encompassing the use of those systems to the control of structures have been reported in the literature (Mitchell et al., 2012; Lin and Zheng, 2012; Muradova et al., 2017). A specific architecture of the Neuro-Fuzzy system is the Adaptive Neuro-Fuzzy Inference System (ANFIS). 
Fuzzy control techniques have been applied to active vibration control of composite structures (Nasser et al., 2012). Nevertheless, it is difficult to define the optimal sets for fuzzy membership functions and fuzzy rules for the synthesis of fuzzy controllers. The definition of these sets usually depends on the experience of the designers and trial-and-error procedures. Consequently, it is necessary the development of novel optimal controllers with the benefits previously exhibited by fuzzy controllers. The intelligent systems based on fuzzy logic and artificial neural networks have been successfully used in several control engineering applications. Thinh et al. (2009) applied an adaptive neuro-fuzzy controller to an ionic polymer-metal composite actuator to cope with performance deterioration produced by nonlinear response effects; the neuro-fuzzy controller helped to improve the dynamic performance of the composite actuator. The present study proposes the active modal control of smart laminated composite structures by using linear quadratic regulator approach and neuro-fuzzy control taking into account the dynamics of the composite structure based on the High-order Shear Deformation Theory (HSDT). The neuro-fuzzy enhances the vibration attenuation performance of the structure based on its learning and adaptive capability, dealing better with nonlinear dynamics and uncertainties than classical controllers such as linear quadratic regulators.

This paper presents the Mixed Theory and its numerical implementation via MEF in the modeling of smart laminated composite structures. The Mixed Theory adopted herein comprises a single layer, in which HSDT theory is used to approximate the mechanical displacement field and the Layerwise theory is used to approximate the electrical degrees of freedom. The results of natural frequencies and sensor/actuator signals are validated with data reported in the literature. The formulation is summarized in the next section, but greater details are reported by Faria (2006). The active control of vibrations is assessed through two control approaches. The first involves the linear quadratic regulator (LQR), which is solved by linear matrix inequalities (LMI) and allows the calculation of the controller's gain, assuming the model parameters include uncertainties. The second consists of employing the Neuro-Fuzzy system associated with ANFIS architecture. The Takagi-Sugenos inference method with the hybrid learning rule is also implemented, which combines the backpropagation algorithm with a decreasing gradient to adjust and optimize the control parameters adequately. The balanced realization method is used to reduce the complexity of the computational method to the vibration modes that are more relevant to the structure. Despite many studies on laminated composite structures theories and in the active vibration control of them, the novelty of the present paper lies in the application of the Neuro-Fuzzy control in the modal domain, taking into account the dynamics of the composite structure based on the High-Order Shear Deformation Theory (HSDT).

\section{MECHANICAL DISPLACEMENT FIELD IN THE MIXED THEORY}

In the Mixed Theory, the mechanical behavior of the structure is modeled through a third and a first-order displacement field when dealing with HSDT and FSDT, respectively. The displacement field considering an HSDT model is as follows:

$$
\begin{array}{r}
u(x, y, z, t)=u_{0}(x, y, t)+z \psi_{x}(x, y, t)+z^{2} \zeta_{x}(x, y, t)+z^{3} \Phi_{x}(x, y, t) \\
v(x, y, z, t)=v_{0}(x, y, t)+z \psi_{y}(x, y, t)+z^{2} \zeta_{y}(x, y, t)+z^{3} \Phi_{y}(x, y, t) \\
w(x, y, z, t)=w_{0}(x, y, t)+z \psi_{z}(x, y, t)+z^{2} \zeta_{z}(x, y, t)
\end{array}
$$

where: $u_{0}, y_{0}$ and $w_{0}$ are the displacements along the coordinate directions $(x, y, z)$ of a material point that belongs to the reference plane $(x, y) ; \psi_{x}, \psi_{y}$ and $\psi_{z}$ are the rotations of the segments that are normal to the reference surface about $\mathrm{x}, \mathrm{y}$ and $\mathrm{z}$ axes, respectively. The functions $\zeta_{x}, \zeta_{y}, \zeta_{z}, \Phi_{x}$ and $\Phi_{y}$ have no evident physical meaning, but may be seen as high-order rotations describing the deformation of a normal line from the reference plane (Chee, 2000).

The mechanical displacement field according to the FSDT model differs from Equation 1 in that it neglects the second and third-order terms of the polynomial expansion, as well as the rotation $\psi_{z}$.

The advantages of the HSDT model over the FSDT are: (i) it is appropriate to the modeling of thin and thick composite plates, (ii) it does not require the use of a correction factor for transverse shear strains $\left(\gamma_{x z}, \gamma_{x z}\right)$, (iii) it does not require functions derived from C1 space (derivative functions) and (iv) it incorporates the transverse normal strain. 
The displacement field brought in by the HSDT model involves eleven unknowns, which may be reduced to only five with the use of FSDT. However, as previously mentioned, the FSDT model requires the correction of transverse shear strains since they are assumed to have a linear distribution along the laminate thickness $z$.

Using definitions from Solid Mechanics together with Equation 1, the strains according to the HSDT model can be expressed as:

$$
\{\varepsilon(x, y, z)\}=[D(z)]\{\hat{u}(x, y)\}
$$

where $\{\varepsilon(x, y, z)\}$ is a vector that includes the bending, membrane and transverse shear terms defined as $\{\varepsilon\}=\left\{\varepsilon_{x x} \varepsilon_{y y} \varepsilon_{z z} \gamma_{y z} \gamma_{z x} \gamma_{x}\right\}^{T}, \quad\{\hat{u}\}$ is the displacement vector with eleven mechanical nodal variables defined as $\{\hat{u}\}=\left\{u_{0} v_{0} w_{0} \psi_{x} \psi_{y} \psi_{z} \zeta_{x} \zeta_{y} \zeta_{z} \Phi_{x} \Phi_{y}\right\}^{T}$ and $[D(z)]$ is the matrix containing powers of the $z$ coordinates and zeros defined by the fields and strain relations (see Appendix A).

The mechanical variables presented in Equations 1 and 2 are converted to their finite element formulation through appropriate shape functions. The finite element considered herein is a plate-type element called Serendipity, with a rectangular shape and on which every edge presents three nodes, totaling eight nodes (Reddy, 2003).

In terms of local coordinates $(\xi, \eta)$, as defined by Reddy (2003), the eight interpolation functions of the aforementioned element (shape functions) can be written as:

$$
\left[\begin{array}{l}
N_{1}(\xi, \eta) \\
N_{2}(\xi, \eta) \\
N_{3}(\xi, \eta) \\
N_{4}(\xi, \eta) \\
N_{5}(\xi, \eta) \\
N_{6}(\xi, \eta) \\
N_{7}(\xi, \eta) \\
N_{8}(\xi, \eta)
\end{array}\right]=\left[\begin{array}{c}
\frac{-1}{4}(1-\xi)(1-\eta)(1+\xi+\eta) \\
\frac{1}{2}(1-\xi)(1+\xi)(1-\eta) \\
\frac{-1}{4}(1+\xi)(1-\eta)(1-\xi+\eta) \\
\frac{1}{2}(1+\xi)(1+\eta)(1-\eta) \\
\frac{-1}{4}(1+\xi)(1+\eta)(1-\xi-\eta) \\
\frac{1}{2}(1-\xi)(1+\xi)(1+\eta) \\
\frac{-1}{4}(1-\xi)(1+\eta)(1+\xi-\eta) \\
\frac{1}{2}(1-\xi)(1+\eta)(1-\eta)
\end{array}\right]
$$

The eleven mechanical variables from the HSDT model are expressed in terms of 88 corresponding nodal mechanical variables as it can be seen from the following equation in the shortened form:

$$
\{\hat{u}(\xi, \eta, t)\}_{n \times 1}=\left[N_{u}(\xi, \eta)\right]_{n \times 8 n}\left\{u_{e}(t)\right\}_{8 n \times 1}
$$

where: $\left[N_{u}(\xi, \eta)\right]$ is the mechanical shape function matrix, $\left\{u_{e}(t)\right\}$ is the nodal displacement vector and $\eta$ is the number of mechanical variables in the theory being employed. Considering the HSDT model, $\eta$ is equal to eleven, whereas it is equal to five considering the FSDT model.

From Equation 4, the mechanical displacement field according to the HSDT model (Equation 1) is expressed in terms of elementary local coordinates as:

$$
\{U(\xi, \eta, z, t)\}_{1 \times 3}=\left[A_{u}(z)\right]_{3 \times n}\left[N_{u}(\xi, \eta)\right]_{n \times 8 n}\left\{u_{e}(t)\right\}_{8 n \times 1}
$$

where: $\{U\}=\left\{\begin{array}{lll}u & v & w\end{array}\right\}^{T}$. The shape functions from Equation (3) are included in the mechanical shape function matrix $\left\{N_{u}(\xi, \eta)\right\}$. The matrix $\left[A_{u}(z)\right]$ is shown in the Appendix A.

The mechanical strains, which are presented in terms of shape functions and nodal mechanical displacements in Equation (2), can be written using Equation (4) in the following form: 


$$
\{\varepsilon(\xi, \eta, z, t)\}_{6 \times 1}=[D(z)]_{6 \times n}\left[N_{u}(\xi, \eta)\right]_{n \times 8 n}\left\{u_{e}(t)\right\}_{8 n \times 1}=\left[B_{u}(\xi, \eta, z)\right]_{6 \times 8 n}\left\{u_{e}(t)\right\}_{8 n \times 1}
$$

where: $[D(z)]$ is described in the Appendix $A$, and $\left[B_{u}\right]$ is the strain-displacement matrix, relating the degrees of freedom of the element with the strain components.

\section{DISTRIBUTION OF THE LINEAR ELECTRIC POTENTIAL ACROSS THE LAYERS}

The linear layerwise technique were used by Saravanos and Heyliger (1995), Chee (2000), Correia et al. (2000), and before them, Robbins and Reddy (1991). In the Mixed Theory, the layerwise formulation will only be applied to the electric potential. According to Chee (2000), the layerwise concept is suitable because the electric voltage is commonly applied across the thickness of the piezoelectric material and the voltage distribution is usually linear, presuming the material is homogenous.

For the purpose of approximating the electric potential in the context of the Mixed Theory, the $z$-coordinate along the plate thickness is uncoupled from the coordinates of the reference surface $(x, y)$, and can be expressed as follows:

$$
\varphi(x, y, z, t)=\sum_{j=1}^{n c+1} L_{j}(z) \varphi_{j}(x, y, t)
$$

where: $L_{j}(z)$ is called layerwise function and $\varphi_{j}(x, y, t)$ are interface functions from the $j$-th interface of the laminated composite with $n_{c}$ layers.

The electric potential acting on each layer may be approximated by a linear function in the direction of the thickness $(z)$. Thus, the electric potential of the $i$-th layer is expressed as:

$\varphi_{\text {layer }(i)}(x, y, z, t)=L_{i d}(z) \varphi_{i}(x, y, t)+L_{i u}(z) \varphi_{i+1}(x, y, t)$

where: $L_{i d}$ and $L_{i u}$ are linear Lagrange interpolation functions of the bottom and top interfaces, respectively, of the $i$-th composite layer. They are defined as:

$L_{i d}(z)=\frac{z-z_{i+1}}{z_{i}-z_{i+1}} ; \quad L_{i u}(z)=\frac{z-z_{i}}{z_{i+1}-z_{i}}$

In the finite element formulation, the $\left(n_{c}+1\right)$ interface functions are expressed in terms of the shape function matrix $\left[\bar{N}_{\varphi}(\xi, \eta)\right]$ and of the corresponding nodal electric potentials $\varphi_{i j} j$ of the vector $\left\{\phi_{e}\right\}$, as denoted below:

$\{\varphi(\xi, \eta, t)\}_{(n c+l) \times 1}=\left[\bar{N}_{\varphi}(\xi, \eta)\right]_{(n c+l) \times 8(n c+l)}\left\{\phi_{e}(t)\right\}_{8(n c+l) \times 1}$

When representing the nodal electric potential $\varphi_{i j}$, the subscript $i$ indicates the number of the layer interface, while the subscript $j$ indicates the local number of the node, varying from 1 to 8 . In the finite element formulation, the electric potential field in terms of local coordinates, considering the $k$-th elementary layer of the $e$-th element, is expressed as:

$$
\begin{aligned}
& \varphi(\xi, \eta, z, t)_{e}^{k}=\left[\begin{array}{llll}
\ldots & L_{k d}(z) & L_{k u}(z) & \cdots
\end{array}\right]_{I \times(n c+l)}\left[\bar{N}_{\varphi}(\xi, \eta)\right]_{(n c+l) \times(8(n c+l))}\left\{\begin{array}{c}
\phi_{1 I}(t) \\
\vdots \\
\phi_{(n+l) l}(t) \\
\vdots \\
\phi_{(n+l) 8}(t)
\end{array}\right\}_{8(n c+l) \times 1}= \\
& {\left[N_{\varphi}(\xi, \eta, z)\right]_{I \times 8(n c+l)}\left\{\phi_{e}(t)\right\}_{8(n c+l) \times I}}
\end{aligned}
$$

where: $\left[N_{\phi}\right]$ is the electric shape function matrix that incorporates the Serendipity shape functions.

Invoking the usual definition of the electric field as the negative gradient of potential along with Equation (11), the expansion of the electric field through the laminate thickness is written in the following form: 


$$
\left\{E(\xi, \eta, z, t)_{e}^{k}\right\}_{3 \times 1}=-\vec{\nabla}\left(\left[N_{\varphi}(\xi, \eta, z)\right]_{1 \times 8(n c+1)}\left\{\phi_{e}(t)\right\}_{8(n c+l) \times 1}\right)=-\left[B_{\varphi}(\xi, \eta, z)_{k}\right]_{3 \times 8(n c+1)}\left\{\phi_{e}(t)\right\}
$$

\section{FORMULATION OF ELEMENTARY MATRICES}

Since a smart structure is generated from active (sensors and actuators) and passive (substrate) materials, the coupling between such elements must be included in the model. This can be achieved through Hamilton's Variational Principle (HVP), which considers every energetic contribution existing in the structure. Based on the procedure developed by Chee (2000), the expressions of matrices obtained on the elementary level are:

$\left[M^{e}\right]=\int_{V e} \rho\left[N_{u}\right]^{T}\left[A_{u}\right]^{T}\left[A_{u}\right]\left[N_{u}\right] d V_{e}$

$\left[K_{u u}^{e}\right]=\sum_{k=1}^{n c} \int_{\xi=-1}^{+1} \int_{\eta}^{+1} \int_{z=z_{k}}^{z_{k+1}}\left(\left[B_{u}\right]^{T}[c]\left[B_{u}\right]\right) J d z d \eta d \xi$

$\left[K_{u \varphi}^{e}\right]=\sum_{k=1}^{n c} \int_{\xi=-1}^{+I} \int_{\eta}^{+l} \int_{z=z_{k}}^{z_{k+l}}\left(\left[B_{u}\right]^{T}[e]^{T}\left[B_{\varphi}\right]\right) J d z d \eta d \xi$

$\left[K_{\varphi \varphi}^{e}\right]=\sum_{k=1}^{n c} \int_{\xi=-1}^{+l} \int_{\eta}^{+1} \int_{z=z_{k}}^{z_{k+1}}-\left(\left[B_{\varphi}\right]^{T}[\chi]\left[B_{\varphi}\right]\right) J d z d \eta d \xi$

where: $\rho$ is the material density, $T$ means transpose, $\left[M^{e}\right]$ is the elementary mass matrix, $\left[K_{u u}^{e}\right]$ is the elementary matrix of elastic stiffness, $\left[K_{u \varphi}^{e}\right]$ and $\left[K_{u \varphi}^{e}\right]=\left[K_{u \varphi}^{e}\right]^{T}$ are elementary matrices of electric-mechanical coupling and $\left[K_{\varphi \varphi}^{e}\right]$ is known as elementary dielectric stiffness matrix. $[c],[e]$ and $[\chi]$ are the matrices of elastic stiffness, piezoelectric stress and electric permittivity constant values, respectively, and $J$ is the Jacobian of the transformation (Reddy, 2003).

The contributions of the superficial electric charges and of the body and surface forces on the elementary level are obtained through the formulation of the virtual work in terms of the shape functions (Equation (3)) and Equations (5) and (12). By substituting these equations into the integral of the virtual work, the following expressions are obtained:

$$
\begin{gathered}
\left\{F_{e}\right\}=\int_{V e}\left[N_{u}\right]^{T}\left[A_{u}\right]^{T}\left\{F^{V}\right\} d V_{e}+\int_{S e}\left[N_{u}\right]^{T}\left[A_{u}\right]^{T}\left\{F^{S}\right\} d S_{e}+\left[N_{u}\right]^{T}\left[A_{u}\right]^{T}\left\{F^{P}\right\} \\
\left\{Q_{e}\right\}=\int_{S e}\left[N_{\varphi}\right]^{T}\left\{Q^{S}\right\} d S_{e}
\end{gathered}
$$

where: $\left\{F_{e}\right\}$ and $\left\{Q_{e}\right\}$ are the vectors of nodal forces and loads, respectively, generalized on the elementary level, $V_{e}$ and $S_{e}$ are respectively defined as elementary volume and area.

Adopting the standard procedure for building the global matrices, the mathematical model of the system can be expressed through the global system of equations of the movement of the coupled electric-mechanical system. This expression is shown below, in which the subscript $g$ indicates global quantities:

$$
\left[\begin{array}{cc}
{\left[M_{g}\right]} & 0 \\
0 & 0
\end{array}\right]\left\{\begin{array}{l}
\left\{\begin{array}{l}
u_{g} \\
\dot{*} \\
\phi_{g}
\end{array}\right\} \\
\}
\end{array}\right\}+\left[\begin{array}{cc}
{\left[K_{u u}\right]} & {\left[K_{u \varphi}\right]} \\
{\left[K_{\varphi u}\right]} & {\left[k_{\varphi \varphi}\right]}
\end{array}\right]\left\{\begin{array}{l}
\left\{u_{g}\right\} \\
\left\{\phi_{g}\right\}
\end{array}\right\}=\left\{\begin{array}{l}
\left\{F_{g}\right\} \\
\left\{Q_{g}\right\}
\end{array}\right\}
$$

\section{MECHANICAL BOUNDARY CONDITIONS}

There can be seen controversial discussions in the scientific literature regarding the applications of boundary conditions to the mechanical degrees of freedom of the high-order terms of the HSDT model. The application of the boundary conditions for simply supported and clamped edges according to Correia et al. (2000), Chee (2000) and Khare et al. (2003) are presented below. 
1. (i) Simply supported edges, with an edge parallel to the $x$-axis:

$$
\begin{aligned}
& u_{0}=\psi_{x}=\zeta_{x}=\Phi_{x}=w_{0}=\psi_{z}=\zeta_{z}=0^{*} \\
& u_{0}=w_{0}=\zeta_{x}=\zeta_{z}=\psi_{z}=0^{*}
\end{aligned}
$$

2. (ii) Simply supported edges, with an edge parallel to the $y$-axis:

$$
\begin{aligned}
& v_{0}=\psi_{y}=\zeta_{y}=\Phi_{y}=w_{0}=\psi_{z}=\zeta_{z}=0^{*} \\
& v_{0}=w_{0}=\zeta_{y}=\zeta_{z}=\psi_{z}=0^{*}
\end{aligned}
$$

3. (iii) Clamped edges:

$$
u_{0}=\psi_{x}=\zeta_{x}=\Phi_{x}=v_{0}=\psi_{y}=\zeta_{y}=\Phi_{y}=w_{0}=\psi_{z}=\zeta_{z}=0
$$

Chee (2000) and Khare et al. (2003); Correia et al (2000).

After defining the boundary conditions, the prescribed mechanical variables are eliminated from the nodal unknowns, which in turn leads to the transformation of the force vectors on the right side of Equation (18).

\section{EQUATIONS OF THE PIEZOELECTRIC SENSORS AND ACTUATORS}

By rearranging Equation (18) and assuming that $\left\{Q_{q}\right\}$ equals zero, the uncoupled dynamic equations in the structural displacements introduced by the actuators and in the voltages measured by the sensors can be expressed as:

$$
\begin{aligned}
& {\left[M_{g}\right]\left\{\ddot{\left.u_{g}\right\}}\right\}+\left[k_{u u}\right]\left\{u_{g}\right\}=\left\{F_{g}\right\}-\left[k_{u \varphi}\right]\left\{\phi_{g}\right\}} \\
& \left\{\phi_{g}\right\}=-\left[k_{\varphi \varphi}\right]^{-1}\left[k_{\varphi u}\right]\left\{u_{g}\right\}
\end{aligned}
$$

An eigenvector problem may be formulated from Equation (19a), whose solution provides the vibration modes as well as the natural frequencies of the system. The equations for static equilibrium are obtained adopting $\left\{u_{g}\right\}$ as zero.

\section{BALANCED REALIZATION}

The reduced model of a system may be obtained through balanced realization, which basically consists of describing the system model in the state space form encompassing the controllability and observability matrices for each state using the Gramians of controllability and observability of the system. The linear transformation that leads the system to this representation is known as balanced transformation. In this procedure, the states associated with small singular values are neglected (Assunção et al (2007); Laub et al., 1987).

Balanced realization is an asymptotically stable realization, in which the Gramians of controllability and observability are equal and diagonal (Conceição et al., 2009). A stable linear-time invariant system can be defined by the following state-space equations:

$$
\begin{aligned}
& \{\dot{x}(t)\}=[A]\{x(t)\}+[B]\{u(t)\} \\
& \{y(t)\}=[C]\{x(t)\}
\end{aligned}
$$

where: $\{x(t)\}$ is the state vector, $[A]$ is the $n \times n$ dynamic matrix, $[B]$ is the $n \times m$ input matrix, $[C]$ is the $s \times n$ output matrix $\{u(t)\}$ is the input force, $\{y(t)\}$ is the output vector, $n$ is the order of the system, $m$ is the number of inputs and $s$ the number of outputs. 
The system is called balanced if the solutions to the following Lyapunov equations (Eq. 22 and 23) are $P=Q=\operatorname{diag}\left(\sigma_{1}, \sigma_{2} \ldots \sigma_{n}\right)$.

$[A][P]+[P][A]^{T}+[B][B]^{T}=0$

$[A]^{T}[Q]+[Q][A]+[C]^{T}[C]=0$

where: $[P]$ and $[Q]$ are the Gramians of controllability and observability, respectively, and $\sigma_{i}, i=1,2, \ldots, n$ are the singular values of the system $\left(\sigma_{1} \geq \sigma_{2} \geq \ldots \geq \sigma_{n} \geq 0\right)$. Every $\sigma_{i}$ is associated with a $x_{i}$ state of the balanced system. $\sigma_{i}$ values quantify the contribution received from $x_{i}$ to the input-output behavior of the system. Since $\sigma_{1} \geq \sigma_{2}$, the system behavior is more affected by $x_{1}$ than by $x_{2}$, due to the fact that the singular values are ordered from the most important to the least important ones. In the balanced realization, the conformity of the reduced model in relation to the full model $(A, B, C)$ depends upon the relation $\sigma_{r} \geq \sigma_{r+1}$, where $r$ is the order of the reduced model. Thus, the reduced state-space system is given by:

$\left\{\dot{x}_{r}(t)\right\}=\left[A_{r}\right]\left\{x_{r}(t)\right\}+\left[B_{r}\right]\left\{u_{r}(t)\right\}$

$\left\{y_{r}(t)\right\}=\left[C_{r}\right]\left\{x_{r}(t)\right\}$

where: $\left\{\dot{x}_{r}(t)\right\}$ is the reduced state vector, $\left[A_{r}\right]$ is the $r \times r$ reduced dynamic matrix, $\left[B_{r}\right]$ is the reduced $r \times r$ input matrix and $\left[C_{r}\right]$ is the reduced $s \times r$ output matrix.

\section{CONTROL APPROACH}

The active modal control is an effective strategy when applied to the control of smart laminated composite structures, since these require a reduced number of sensors and actuators.

The smart laminated composite structure described in Equation (18) has been represented in the state space form during the simulations, in accordance with Equations (20) and (21). The controllers then used the states to determine the control force.

The Kalman estimator, which can estimate the states using noisy measurement signals, was employed to determine the nodal states required by the controllers. Further details concerning the Kalman estimator may be found in the scientific literature (e.g. Welch e Bishop, 1995; Anderson e Moore, 1979; Durbin e Koopman, 2002). The referred estimator can be represented by Equation (26):

$$
\left\{\dot{x}_{r}(t)\right\}=\left[A_{r}\right]\left\{x_{r}(t)\right\}+\left[B_{r}\right]\{u(t)\}+[L]\{\delta(t)-\bar{\delta}(t)\}
$$

where: $[L]$ is the gain matrix, $\delta(t)$ the displacement vector and $\bar{\delta}(t)$ the estimated displacement vector.

\section{LINEAR QUADRATIC REGULATOR USING LINEAR MATRIX INEQUALITIES}

The linear quadratic regulator is an optimal control that provides a methodology to control the feedback gain, assuring satisfactory levels of stability to the system. In the context of the present contribution, the LQR is designed so that the performance index minimization leads to the optimization of previously set physical quantities (Ogata, 2010).

The feedback control gain can be determined through the performance index minimization given by Equation (27):

$$
J=\int_{0}^{\infty}\{x(t)\}^{T}\left[Q_{l q r}\right]\{x(t)\}+\{u(t)\}^{T}\left[R_{l q r}\right]\{u(t)\} d t
$$


where: $\left[Q_{l q r}\right]$ is the positive-definite Hermitian (or positive semi-definite) or real symmetric matrix that weights each state, $\left[R_{l q r}\right]$ is the positive-definite or real symmetric matrix that weights the energy cost of the controller (Simões et al, 2007).

The controller gain obtained through LMI involves the assumption that the model parameters involve uncertainties. Erkus and Lee (2004) present the LQR controller solved by LMI, showing that the LQR-LMI problem is described by:

$$
\begin{gathered}
\min _{X, P_{l m i}, X_{l m i}} \operatorname{tr}\left(\left[Q_{l q r}\right]\left[P_{l m i}\right]\right)+\operatorname{tr}\left(\left[X_{l m i}\right]\right)+\operatorname{tr}\left(\left[Y_{l m i}\right] N\right)+\operatorname{tr}\left([N]^{T} Y_{l m i}{ }^{T}\right) \\
{[A][P]-[B]\left[Y_{l m i}\right]+[P][A]^{T}-\left[Y_{l m i}\right]^{T}[B]^{T}+\left[B_{w}\right]\left[B_{w}\right]^{T}<0} \\
{\left[\begin{array}{cc}
{\left[X_{l m i}\right]} & {\left[R_{l q r}\right]^{\frac{1}{2}}\left[Y_{l m i}\right]} \\
{\left[Y_{l m i}\right]^{T}\left[R_{l q r}\right]^{\frac{l}{2}}} & {\left[P_{l m i}\right]}
\end{array}\right]>0}
\end{gathered}
$$

where: $\mathrm{N}$ is the noise position vector, $\left[X_{l m i}\right]$ and $\left[Y_{l m i}\right]$ are LMI solutions, $\operatorname{tr}\left(0\right.$ denotes the matrix trace and $\left[B_{w}\right]$ the disturbance noise.

Equation (28) was rearranged to minimize the performance index given by Equation (27) and to include the noise influence on the process. After solving Equation (28) considering the constraints from Equation (29), the controller gain is:

$$
[G]=\left[Y_{l m i}\right]\left[P_{l m i}\right]^{-1}
$$

\section{DESIGN OF THE ROBUST CONTROLLER USING LMI}

The key advantage of using LMI lies in the fact that it is possible to include numerous specifications, such as the stability degree, decay rate, input limitation for the actuators and bounds on the output peaks. Once again, it is possible to assume that the model parameters involve uncertainties (Ogata, 2010).

$\mathrm{LMI}$ is a very useful tool for mathematical problems involving restrictions, in which the parameters vary according to a range of values. Assunção et al. (2007) presented the design of the robust controllers employed herein. A system of polytopic uncertainties is said to be stable if there exist $[X]$ and $[G]$ such that the following LMI is feasible:

$$
\left[A_{i}\right][X]\left[B_{i}\right][G]+[X]\left[A_{i}\right]^{T}-[G]^{T}\left[B_{i}\right]^{T}<0
$$

$[X]>0$

where: $i=1,2, \ldots, m$ ( $m$ is the number of uncertainties) and $[X]$ the LMI solution.

The robust control using LQR has been applied to Equation (28), and the constraints (Equation (29)) have been organized in the way given by Equation (31).

\section{NEURO-FUZZY CONTROL}

Neural networks are formed by a collection of elements called neurons, which are responsible for the execution of information processing. In conjunction with the Fuzzy logic, it increases the efficiency of this controller. The Neuro-Fuzzy systems can conveniently deal with the complexity, non-linearity, and uncertainties of a model.

The ANFIS Neuro-Fuzzy system adopts a Fuzzy inference system based on the model developed by Takagi-Sugeno conjointly with neural networks. Using a training process, it is possible to tune the Fuzzy inference system to generate the desired set of input-output data.

The ANFIS architecture has five layers of neurons, as depicted in Figure 1, each having the function delineated below (Jang, 1993). 


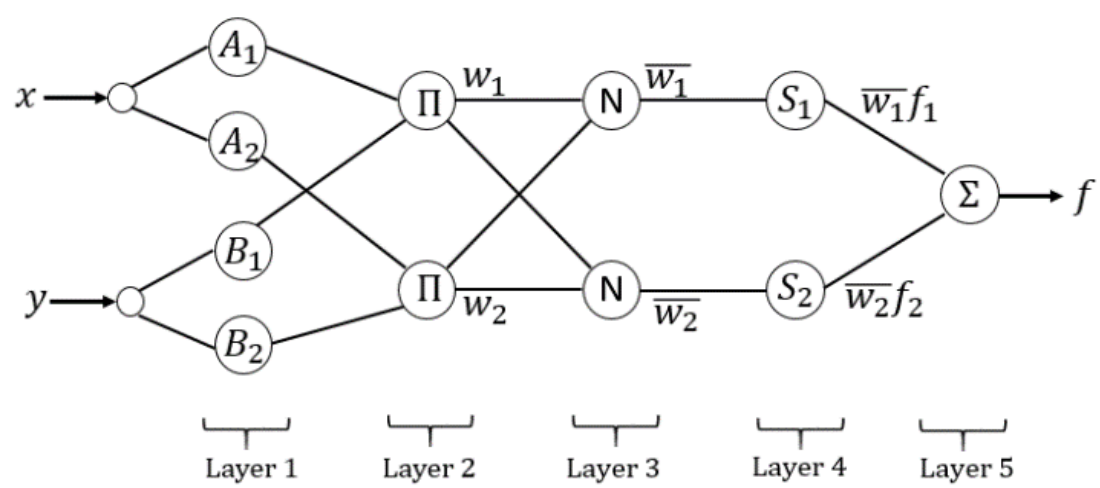

Figure 1: ANFIS Architecture. (Adapted from Jang, 1993).

In layer 1 , the degree of membership is calculated based on the inputs $x$ and $y$ (displacement and velocity, respectively) of the membership function. The generalized bell-shaped (gbell) membership function was chosen, which is expressed as follows:

$$
O_{1, i}=\mu_{A i}(x)=\frac{1}{1+\left|\frac{x-c_{i}}{a_{i}}\right|^{2 b_{i}}}
$$

where: $x$ is the input value, $a, b$ and $c$ are parameters of the membership function.

Each neuron in layer 2 executes the operation of intersecting the membership functions, according to Equation (33). The output of this intersection is the firing strength of each rule $\left(w_{i}\right)$ :

$O_{2, i}=w_{i}=\mu_{A i}(x) \mu_{B i}(x), i=1,2$

In the third layer, the membership functions are normalized. Each node represents a calculation of the ratio between the $i$-th rule's firing strength and the sum of all rules' firing strength. This process can be expressed as follows:

$O_{3, i}=\overline{w_{i}}=\frac{w_{i}}{w_{1}+w_{2}}, i=1,2$

In the fourth layer, the parameters are referred to as consequent parameters of the rule involving the activation function, which assumes the Sugeno's first-order model:

$O_{4, i}=\bar{w}_{i} f_{i}=\bar{w}_{i}\left(p_{i} x+q_{i} y+r_{i}\right), i=1,2$

where: $p_{i}, q_{i}$ and $r_{i}$ are the consequent parameters of the corresponding node $i$ to be adjusted (linear combinations of the input values).

The last architecture layer calculates the defuzzified output of the system, according to the following equation:

$O_{5, i}=\sum_{i} \bar{w}_{i} f_{i}=\frac{\sum w_{i} f_{i}}{\sum w_{i}}, i=1,2$

The parameters in every layer of neurons are updated in consonance with the training data and the learning process. The hybrid learning algorithm that has been adopted herein combines the gradient descent method for computing the premise parameters and the least square method for estimating or identifying the consequent parameters. 


\section{NUMERICAL APPLICATIONS}

\subsection{Validation of The Mixed Theory Formulation}

Three different numerical applications have been implemented in the Matlab programming platform to illustrate the use and validate the formulation of the HSDT model associated with the Layerwise Theory, originating the Mixed Theory applied to the modeling of laminated composite structures via MEF.

The first numerical simulation aims to demonstrate the accuracy of the Mixed Theory in the modeling of a laminated composite plate consisting of piezoelectric actuators. The second simulation has the same goal, although considering a polyvinylidene fluoride (PVDF) beam constituted by piezoelectric sensors. In the third numerical application, the natural frequencies and vibration modes of laminated composite plates are obtained to demonstrate the accuracy of the Mixed Theory in the modeling of thin and thick composite structures.

The purpose of the first investigation is aimed at obtaining the deflections resulting from an electric voltage application on the superior and inferior piezoelectric inserts of a cantilever composite plate, also known as indirect effect. This test is undertaken on a plate with a stratification sequence of $[0 \% /+45 \% /-450]_{s}$ and subjected to a $100 \mathrm{~V}$ voltage through the thickness of each piezoelectric ceramic.

The ASA/3501 graphite/epoxy cantilever plate, depicted in Figure 2, has a length of $292 \mathrm{~mm}$, a width of $152 \mathrm{~mm}$ and is composed by 6 layers, each $0.83 \mathrm{~mm}$ thick.

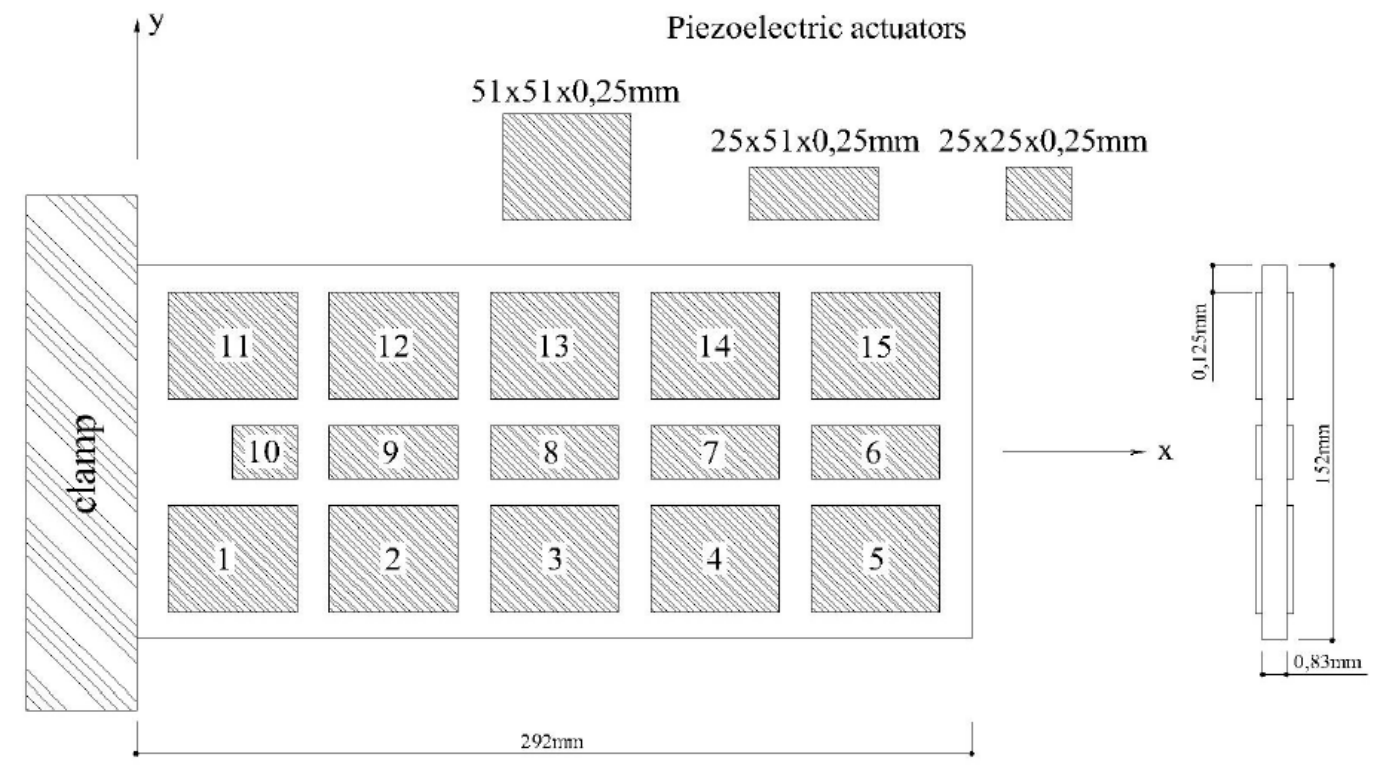

Figure $\mathbf{2}$ - Cantilever composite plate with piezoelectric actuators attached to its surface.

Fifteen G1195 piezoelectric ceramic inserts, each $0.25 \mathrm{~mm}$ thick, are fixed on both inferior and superior faces of the composite plate. The piezoelectric inserts from the first and third lines of actuators have dimensions of $51 \times 51 \mathrm{~mm}^{2}$, while those from the intermediate line have dimensions of $25 \times 51 \mathrm{~mm}^{2}$. The inserts of the piezoelectric element whose assigned number is 10 have dimensions of $25 \times 25 \mathrm{~mm}^{2}$. The plate was discretized into a $12 \times 7$ mesh with 8 layers, which include the layers containing the piezoceramic inserts. Table 1 lists the parameters that define the elastic stiffness, $C_{i}$, the piezoelectric strain constants of the piezoceramic, $d_{i}$, and the electric permittivity, $\chi_{i}$; with $i$ being the correspondent coefficients of the parameters in the model.

Figure 3 compares the numerical results obtained with those from Crawley and Lazarus (1991), Koconis et al. (1994), Lima Jr. (1999) and Chee (2000). The figure shows the deflection values concerning the nodes positioned along the central axis of the composite plate, obtained through Equation (19a) and excluding the effects of the mass matrix. 


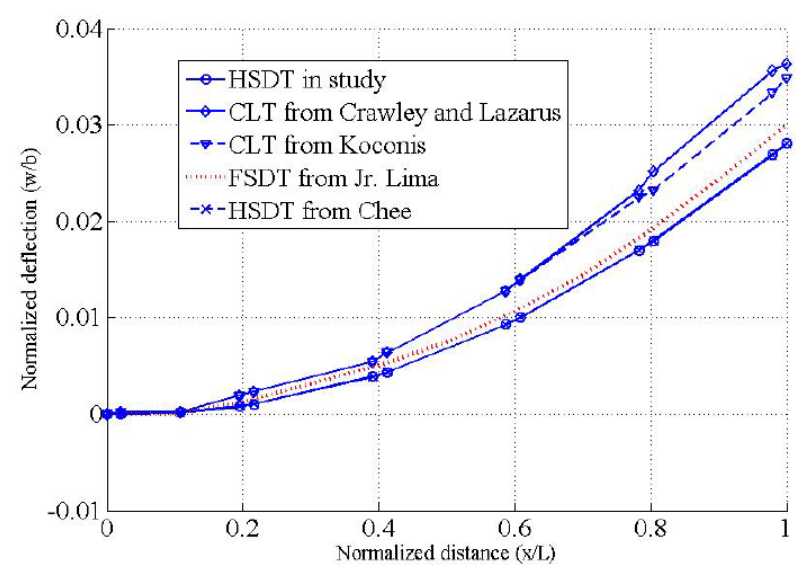

Figure 3 - Normalized deflection of the cantilever composite plate with piezoelectric inserts subjected to a $100 \mathrm{~V}$ voltage

Table 1 Cantilever Composite Plate and piezoelectric actuator parameters

\begin{tabular}{|c|c|c|c|c|}
\hline Element & Symbol & Unite & Value & Equivalence \\
\hline \multirow{6}{*}{$\begin{array}{c}\text { ASA/3501 } \\
\text { graphite/epoxy }\end{array}$} & $C_{11}$ & $\mathrm{GPa}$ & 173.6 & - \\
\hline & $C_{22}$ & $\mathrm{GPa}$ & 7.61 & $C_{22}=C_{33}$ \\
\hline & $C_{12}$ & GPa & 2.48 & $C_{12}=C_{13}$ \\
\hline & $C_{23}$ & GPa & 2.31 & - \\
\hline & $C_{44}$ & GPa & 1.38 & - \\
\hline & $C_{55}$ & GPa & 4.45 & $C_{55}=C_{66}$ \\
\hline \multirow{12}{*}{$\begin{array}{l}\text { G1195 piezoelectric } \\
\text { ceramic }\end{array}$} & $C_{11}$ & GPa & 145.9 & - \\
\hline & $C_{22}$ & GPa & 11.38 & $C_{22}=C_{33}$ \\
\hline & $C_{13}$ & GPa & 4.703 & - \\
\hline & $C_{23}$ & $\mathrm{GPa}$ & 4.295 & - \\
\hline & $C_{44}$ & $\mathrm{GPa}$ & 4 & - \\
\hline & $C_{55}$ & $\mathrm{GPa}$ & 6 & $C_{55}=C_{66}$ \\
\hline & $d_{31}$ & $\mathrm{Pm} / \mathrm{V}$ & - 264.16 & - \\
\hline & $d_{32}$ & $\mathrm{Pm} / \mathrm{V}$ & - 264.16 & - \\
\hline & $d_{33}$ & $\mathrm{Pm} / \mathrm{V}$ & 374 & - \\
\hline & $d_{15}$ & $\mathrm{Pm} / \mathrm{V}$ & 584 & $d_{15}=d_{24}$ \\
\hline & $\chi_{11}$ & $\mathrm{~F} / \mathrm{m}$ & $1.53 \times 10^{-8}$ & $\chi_{11}=\chi_{22}$ \\
\hline & $\chi_{33}$ & $\mathrm{~F} / \mathrm{m}$ & $1.50 \times 10^{-8}$ & - \\
\hline
\end{tabular}

It should be noted that the works of Crawley and Lazarus (1991) and Koconis et al. (1994) adopt the Classical Laminate Theory (CLT), which neglects the effects of the transverse shear and uses the concept of induced strain, disregarding the influence of the piezoelectric elements on the structure mass and stiffness. Thus, when HSDT and FSDT are compared to the preceding models, it is possible to note that the first has an additional stiffness introduced by the piezoelectric materials, which might explain the lower values of deflection depicted in Figure 3 . The results from the models implemented by Chee (2000) and Lima Jr. (1999) are close to those obtained in this study. The HSDT model from Chee (2000) provides a normalized deflection of 0.028, in agreement with that obtained herein, whereas the FSDT model from Lima Jr. (1999) provides a deflection of 0.030. Lima Jr. (1999), however, does not apply a correction factor to the transverse shear strains in the implemented FSDT model, as confirmed by Reddy JN (2003). The absence of such factor generated an approximate discrepancy of $6.667 \%$ in the normalized deflection throughout the thickness of the composite plate compared with the previous two HDST models. 
The next numerical test is based upon the experiment conducted by Tzou and Tseng (1990), which was applied by several other authors such as Hwang and Park (1993), Detwiler et al. (1995), Tzou and Ye (1996), Correia et al. (2000) and Chee (2000) to validate the direct effect derived from the piezoelectric sensors. Such effect consists in the capacity certain materials have of generating an electrical charge that is proportional to the force applied externally.

The structure is an isotropic cantilever beam, composed of two PVDF layers that have opposite polarization directions. The dimensions of this structure are shown in Figure 4.

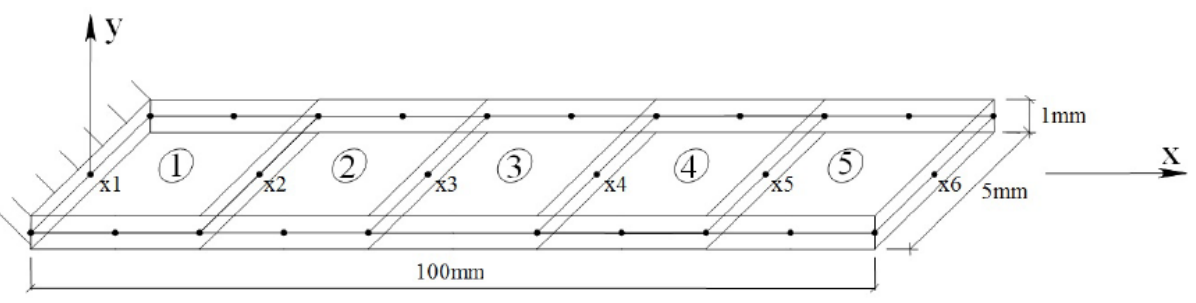

Figure 4 - Piezoelectric cantilever beam.

The mechanical and piezoelectric properties of the employed PVDF beam are: $E_{1}=E_{2}=E_{3}=2 \mathrm{GPa}, G_{12}=G_{13}=G_{23}=1 \mathrm{GPa}$, $v_{12}=v_{13}=v_{23}=0, e_{31}=e_{32}=0.0460 \mathrm{C} / \mathrm{m}^{2}$ and $\chi_{11}=\chi_{22}=\chi_{33}=1.062 \times 10^{-8} \mathrm{~F} / \mathrm{m}$. The Poisson ratio is considered zero to simulate plane stress condition. The piezoelectric beam is modeled using a total of 5 equal elements, each consisting of two discrete layers.

The free end of the beam was subjected to a deflection of $10 \mathrm{~mm}$ applied to node six. The voltages in the sensors are obtained calculating the difference between the top and bottom layers of the piezoelectric beam.

Figure 5 illustrates the voltage difference (electric potential), using Equation (19b), along the five elements in which the beam was discretized.

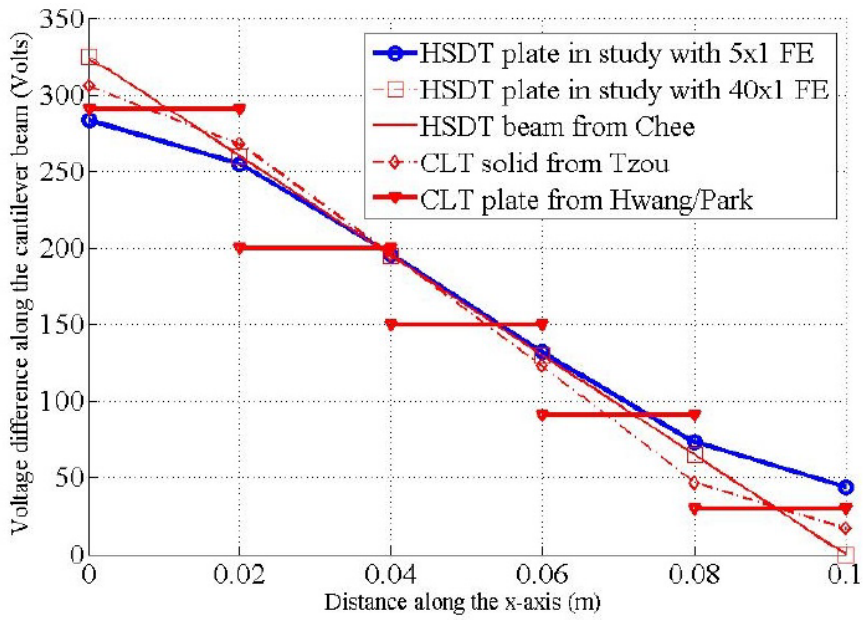

Figure 5. Voltage difference along the length of the piezoelectric cantilever beam acting as a voltage sensor.

Several models were used to compare the results with those obtained through the HSDT model explored herein: the CLT plate model from Hwang and Park (1993) considering five separate electrode components distributed along the length of the beam, each having a constant voltage equipotential surface represented by one g.d.l per component. In fact, due to conductivity, if two or more piezoelectric components are adjacent to each other, their voltage correspond to the median values (Detwiler et al., 1995).

The distribution of electric potential provided by the HSDT formulation presented in this study, as well as by the works of Correia et al. (2000), the CLT solid model of Tzou et al. (1994) and the HSDT beam of Chee (2000) do not exhibit the discontinuities that were verified in the model of Hwang and Park (1993) and allows voltage gradients along the $x$ direction of the cantilever beam. In the present model, the distribution of electric potential is continuous due to the continuity of the electric potential at the nodes of adjacent elements.

It can be noted, from Figure 5, that the discrepancy found in the distribution of electric potential is higher at the ends of the piezoelectric beam than that observed by the previously cited authors. Such discrepancy was also observed in the models studied by Ganapathi et al. (2004), who attributed this variation to the existence of a tridimensional stress 
state in the vicinity of those areas, differently from the model herein employed, which is based upon bidimensional approximations. In certain cases, changes in the trend of potential and stress variations along the length of the beam are observed in the proximity of both free and supported ends due to eventual boundary effects.

It was verified that, whenever the beam is discretized in a higher number of finite elements along the $x$-axis (40x1 elements - Figure 5), a trend of linear variation in the electric potential is observed along the length of the beam. The high-order models in the works of Chee (2000) and Correia et al. (2000) also noticed this. In addition, as it can be depicted from Figure 5, there is an electric potential difference of $323 \mathrm{~V}$ between the free (0V) and clamped ends of the piezoelectric cantilever beam for a $10 \mathrm{~mm}$ deflection, which is very close to the potential difference obtained by Chee (2000) and Correia et al. (2000), 325V, who employed HSDT in the beam discretization.

In the third numerical application the modal analysis of the square laminated composite plate is performed. The plate is simply supported and consists of five layers [p/0/90/0/p], two of them being piezoelectric (p).

Two types of plates have been considered: a thin plate, for which the width/thickness relation $(h / a)$ is 0.02 and a thick plate with $h / a=0.25$.

The laminate is formed by two continuous PZT-4 layers, each $0.1 \mathrm{~h}$ thick, attached to both superior and inferior faces of the graphite/epoxy core [0/90/0]. Each layer of the core is $0.267 \mathrm{~h}$ thick.

To predict the natural frequencies, the plate was discretized into a uniform mesh of $8 \times 8$ elements in its plane. The results obtained according to the HSDT formulation proposed herein are compared to the exact solution from the Theory of Elasticity and the numerical solution through finite elements, both reported by Correia et al. (2000). Their work employed a HSDT formulation including the single layer theory for the approximation of the mechanical displacement field and included rectangular Lagrange elements with nine nodes.

The elastic properties of the graphite/epoxy layers of the composite laminate are: $E_{1}=132.38 \mathrm{GPa}, E_{2}=E_{3}=10.76 \mathrm{GPa}$, $G_{12}=3.61 \mathrm{GPa}, G_{13}=G_{23}=5.65 \mathrm{GPa}, v_{12}=v_{13}=0.24 v_{23}=0.49$. The elastic and piezoelectric constants of the PZT-4 layers are: $E_{1}=E_{2}=81.3 \mathrm{GPa}, \quad E_{3}=64.5 \mathrm{GPa}, G_{12}=G_{13}=25.6 \mathrm{GPa}, \quad G_{23}=30.6 \mathrm{GPa}, \quad v_{12}=0.22, v_{13}=v_{23}=0.43, \quad \rho=7600 \mathrm{~kg} / \mathrm{m}^{3}$, $d_{31}=d_{32}=-122 \times 10^{-12} \mathrm{C} / \mathrm{N}, d_{33}=-285 \times 10^{-12} \mathrm{C} / \mathrm{N}, e_{33}=2100 \times \varepsilon_{0}$ and $\varepsilon_{0}=8.85 \times 10^{-12} \mathrm{~F} / \mathrm{m}$.

The density $\rho$ of each layer is assumed to be $1 \mathrm{~kg} / \mathrm{m}^{3}$ and the boundary conditions of the sides parallel to the $x$-axis are expressed by $u_{0}=w_{0}=\zeta_{y}=\zeta_{z}=\psi_{z}=0$, whereas those of the sides parallel to the $y$-axis are expressed by $\mathrm{v}_{0}=v_{0}=w_{0}=\zeta_{y}=\zeta_{z}=\psi_{z}=0$.

Tables 2 and 3 present the values of the first five natural frequencies, which have been normalized with the use of the following equation:

$$
\left(f_{i}\right)_{\text {norm }}=f_{i} \frac{a^{2}}{h \sqrt{\rho}} 10^{3}
$$

where: $i=1,2, \ldots 5$.

Two types of electric boundary conditions are considered in this case: concerning closed (CC) and open circuit (OC). In the first condition, the electric potential is forced to remain zero (grounded piezoelectric inserts), while in the second condition the electric potential remains free (electrical displacements are zero).

Table 2. Values of the first five normalized natural frequencies considering open circuit conditions.

\begin{tabular}{cccc}
\hline Theory & Vibration & h/a=0.02 & h/a=0.25 \\
\hline & Mode & {$[\mathrm{Hz}]$} & {$[\mathrm{Hz}]$} \\
Exact Solution & 1 & 245.941 & 145.339 \\
HSDT from Correia et al. (2000) & 1 & 250.497 & 142.817 \\
& 2 & 583.185 & 183.491 \\
& 3 & 695.697 & 199.299 \\
HSDT herein presented & 4 & 980.361 & 267.975 \\
& 5 & 1145.406 & 270.125 \\
& 1 & 244.993 & 144.506 \\
& 2 & 562.074 & 205.842 \\
& 3 & 695.116 & 205.867 \\
\end{tabular}


Table 3. Values of the first five normalized natural frequencies considering closed circuit conditions.

\begin{tabular}{cccc}
\hline Theory & Vibration & h/a=0.02 & h/a=0.25 \\
\hline & Mode & {$[\mathrm{Hz}]$} & {$[\mathrm{Hz}]$} \\
Exact Solution & 1 & 245.942 & 145.377 \\
HSDT from Correia et al. (2000) & 1 & 230.461 & 139.683 \\
& 2 & 520.384 & 183.212 \\
& 3 & 662.915 & 198.998 \\
HSDT herein presented & 4 & 908,459 & 263.631 \\
& 5 & 1022.091 & 266.305 \\
& 1 & 231.875 & 139.635 \\
& 2 & 526.567 & 205.839 \\
& 3 & 668.023 & 205.867 \\
\end{tabular}

From the results shown in Tables 2 and 3, the influence of the piezoelectric effect on the values of natural frequencies can be noticed, since the difference between the values considering closed and open circuit conditions is not significant.

Concerning the first vibration mode (known as the fundamental frequency) in both thick ( $\mathrm{h} / \mathrm{a}=0.25)$ and thin $(\mathrm{h} / \mathrm{a}=0.02)$ plates, the HSDT formulation presented in this study and that from Correia et al. (2000) demonstrates relatively close results concerning the exact solution derived from the Theory of Elasticity (TE). The prediction of the fundamental frequency can converge to values both higher or lower than those obtained through the exact solution, depending upon the electric boundary conditions (CC or OC) as well as the thickness of the composite plate (which is, in turn, the most influencing factor on the values of natural frequencies). Tables 2 and 3 show that the first five natural frequencies increase with the vibration mode, regardless the electric boundary of the piezoelectric element. On the other hand, under a CC condition the values of natural frequencies become slightly lower due to the absence of an electric-mechanical coupling.

Furthermore, according to Tables 2 and 3, the values of the fundamental frequency obtained through the implemented HSDT model for both thin and thick plates under OC electric boundaries are closer to the exact TE solution than the results from Correia et al. (2000). The percentual errors between the results from the HSDT model proposed and the exact solution are 0.386 and $0.599 \%$ for thick and thin plates, respectively. The same percentual errors rise to 1.852 and $1.761 \%$ when the model suggested by Correia et al. is used. If, nonetheless, a CC electric boundary is adopted, the fundamental frequency of the plates modeled via the proposed HSDT model demonstrates greater errors when compared with the exact solution (5.719 and 3.925\% for thick and thin plates, respectively). In comparison with Correia et al. (2000) under CC conditions, the errors between the model results and the exact solution are 0.614 and $0.034 \%$, for thin and thick plates, respectively.

The observed differences are higher considering open circuit conditions. The differences between the obtained results in both HSDT formulations may be associated with the use of distinct elements. Correia et al. (2000) worked with a 9-node Lagrangian element, differently from the present formulation that incorporated an 8-node Serendipity element.

Figure 6 shown below, illustrate the five first vibration modes of the thick laminated composite plate whose width/thickness relation $(h / a)$ is 0.25 , considering closed circuit condition. The vibration modes for strains were all normalized through the modal mass matrix.

\subsection{Active Control of Smart Composite Structures}

The laminated composite beam studied in this work is presented in Figure 7 and has a length of 306 mm, width of $25.5 \mathrm{~mm}$ and thickness of $1 \mathrm{~mm}$. The beam is formed by five graphite/epoxy layers, each $0.2 \mathrm{~mm}$ thick, with orientations

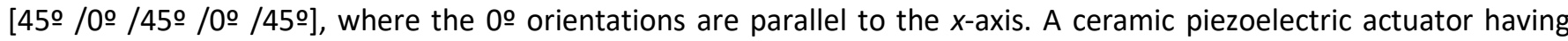
dimensions of $45.9 \times 25.5 \mathrm{~mm}^{2}$ and $1 \mathrm{~mm}$ thick is attached to the superior surface of the beam, $1 \mathrm{~mm}$ away from the clamp. The elastic stiffness constants of the laminated composite beam made from graphite/epoxy (ASA/3501) and PZT layers are listed in table 4 . With $\varepsilon_{0}=8.85 \times 10^{-12} \mathrm{~F} / \mathrm{m}$.

The boundary conditions in control experiments correspond to the Cantilever composite beam of Figure 7, i.e., clamped-free beam. The beam is at rest in the initial condition. A unit impulse excitation force, $F(t)$, was applied to point (II) at the beginning of the experiment. 


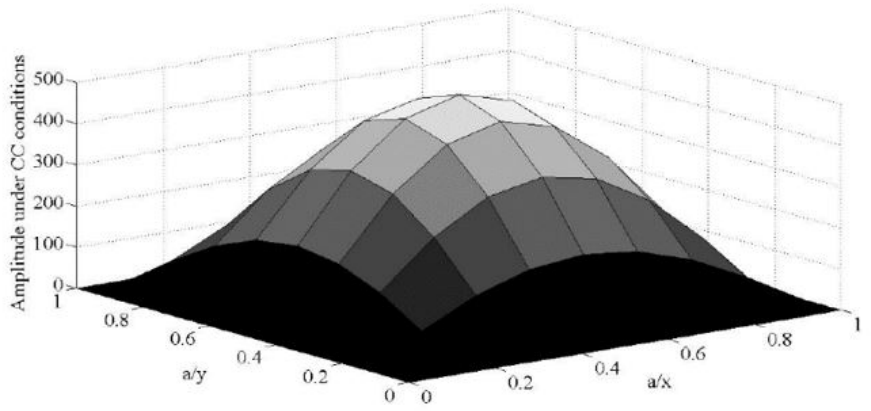

(a) $1^{\text {st }}$ vibration mode $\left(f_{\text {norm }}\right)_{1}=139.635 \mathrm{~Hz}$

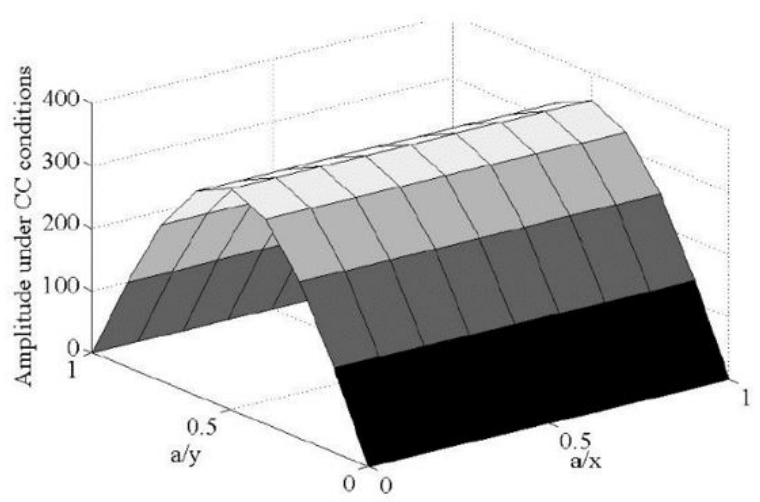

(c) $3^{\text {rd }}$ vibration mode $\left(f_{\text {norm }}\right)_{3}=205.867 \mathrm{~Hz}$

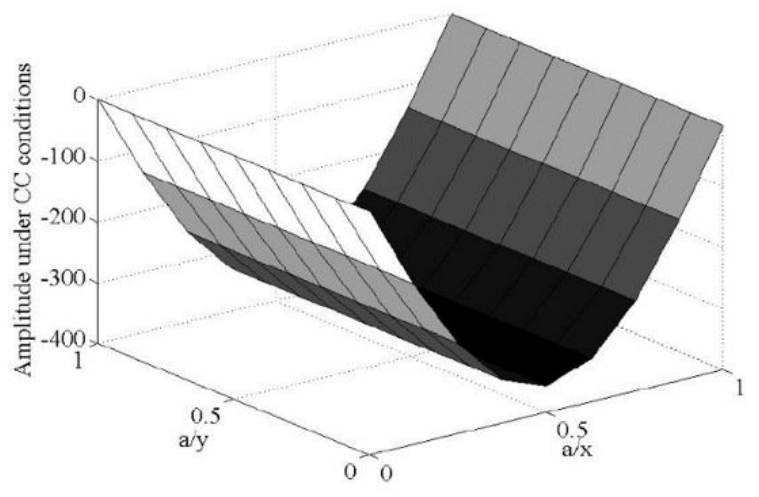

(b) $2^{\text {nt }}$ vibration mode $\left(f_{\text {norm }}\right)_{2}=205.839 \mathrm{~Hz}$

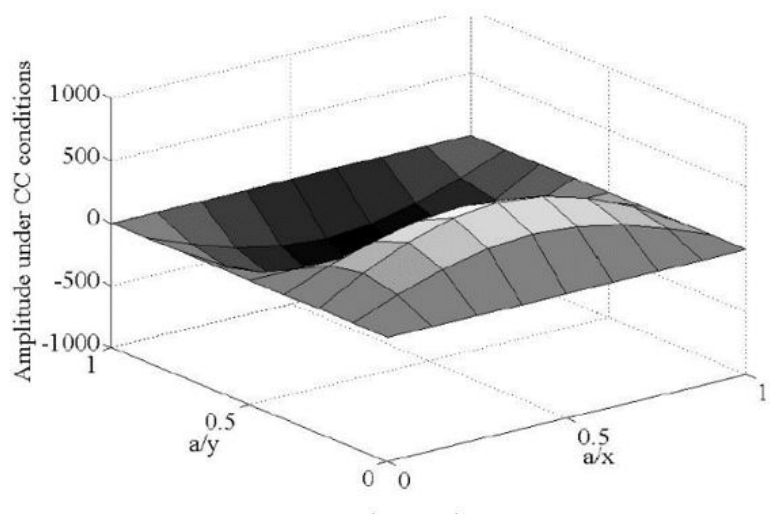

(d) $4^{\text {th }}$ vibration mode $\left(f_{\text {norm }}\right)_{4}=263.141 \mathrm{~Hz}$

Figure 6 - Vibration mode: CC conditions.

The structure responses in time-domain were captured at point (I). The piezoelectric actuator is connected to an active control system and, thus, the vibration amplitudes must be minimized over time. Band-limited white noise is superimposed over the calculated displacements aiming a better approximation of actual behavior.

Two cases of active vibration control have been analyzed: (a) Robustness analysis considering the variation in the dynamic matrix of the structure model $[A]+[\Delta A]$ and (b) robustness analysis considering the variation in the dynamic matrix of the Kalman Estimator $[A]+\left[\Delta A_{r}\right]$.

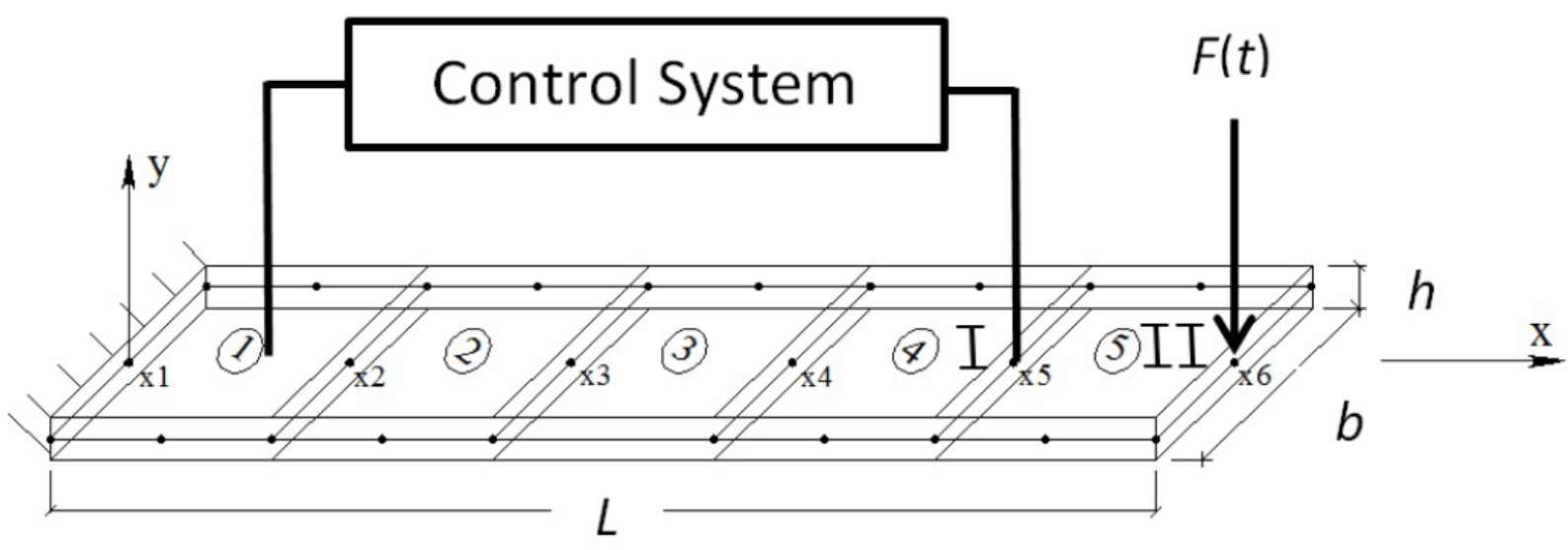

Figure 7 - Cantilever composite beam. 
Table 4 Cantilever Composite beam of the Figure 7

\begin{tabular}{|c|c|c|c|c|}
\hline Element & Symbol & Unite & Value & Equivalence \\
\hline \multirow[t]{7}{*}{ ASA/3501 } & $C_{11}$ & $\mathrm{GPa}$ & 173.6 & - \\
\hline & $C_{22}$ & GPa & 7.61 & $C_{22}=C_{33}$ \\
\hline & $C_{12}$ & GPa & 2.48 & $C_{12}=C_{13}$ \\
\hline & $C_{23}$ & $\mathrm{GPa}$ & 2.31 & - \\
\hline & $C_{44}$ & $\mathrm{GPa}$ & 1.38 & - \\
\hline & $C_{55}$ & GPa & 4.45 & $C_{55}=C_{66}$ \\
\hline & Density & $\mathrm{Kg} / \mathrm{m}^{3}$ & 1.578 & - \\
\hline \multirow[t]{8}{*}{ PZT Layer } & $C_{11}$ & $\mathrm{GPa}$ & 102.23 & $C_{22}=C_{33}$ \\
\hline & $C_{12}$ & $\mathrm{GPa}$ & 5.035 & $C_{13}=C_{23}$ \\
\hline & $C_{44}$ & GPa & 2.594 & $C_{55}=C_{66}$ \\
\hline & $e_{31}$ & GPa & -18.300 & - \\
\hline & $e_{32}$ & GPa & -9.013 & $e_{32}=e_{33}$ \\
\hline & $\chi_{11}$ & $\mathrm{~F} / \mathrm{m}$ & $1800 \times \varepsilon_{0}$ & $\chi_{22}=\chi_{33}$ \\
\hline & $\chi_{33}$ & $\mathrm{~F} / \mathrm{m}$ & $1.50 \times 10^{-8}$ & - \\
\hline & Density & $\mathrm{Kg} / \mathrm{m}^{3}$ & 7.7 & - \\
\hline
\end{tabular}

The variation applied to case (a) corresponds to eventual changes in the structure, whereas the variation applied to case (b) is associated with uncertainties from the system identification. Only non-parametric uncertainties were considered, with a level of $10 \%$ around the nominal value. The samples of the uncertainty term $\Delta$ are determined through Monte Carlo simulations combined with the Latin hypercube for a set of 100 samples.

The balanced realization was utilized in order to reduce the model to the first structure mode, in which the system was observable and controllable. Table 5 presents the two uncertainty configurations of the model (matrix $A_{i}$ ). These models were used to determine the robust controllers.

Table 5. Uncertainty model.

\begin{tabular}{cccc}
\hline & \multicolumn{3}{c}{ Mode 1} \\
\cline { 2 - 4 } & $A_{i}$ & $-\mathbf{1 0 \%}$ & $\mathbf{+ 1 0 \%}$ \\
\hline 1 & $\mathrm{X}$ & - & $\mathrm{X}$ \\
\hline
\end{tabular}

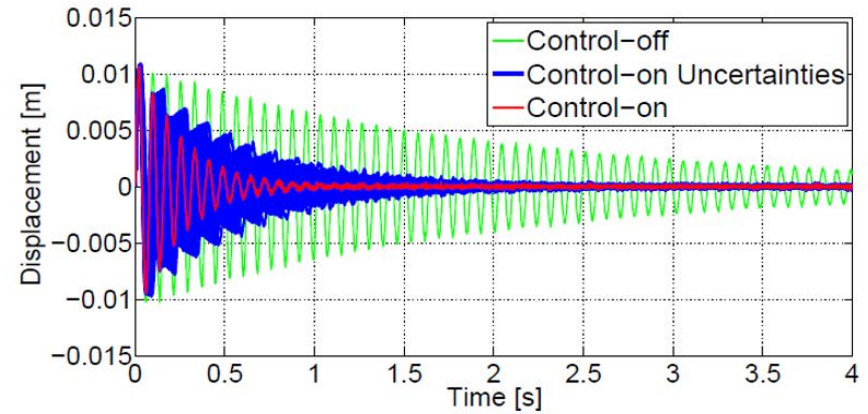

(a) LQR/LMI controller (case a)

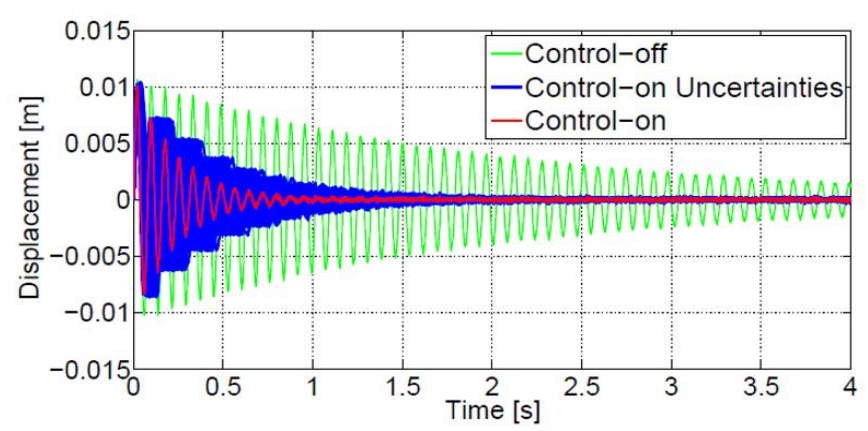

(b) Neuro-Fuzzy controller (case a)

Figure 8. Impact responses to the LQR/LMI, and Neuro-fuzzy controllers for case (a).

Figures 8 to 10 present the results considering the uncertainties in the dynamic matrix of the structure model (case a). These results were divided into two groups, where the first comprises the results from the LQR controller solved by LMI (Figures $8(\mathrm{a}), 9(\mathrm{a})$ and 10(a)) and the second comprises the results from the Neuro-Fuzzy controller (Figures $8(\mathrm{~b})$, $9(b)$ and $10(b))$. 
The results of the structure displacements when it is being subjected to an impact, depicted in Figures $8(\mathrm{a})$ and $8(\mathrm{~b})$, show that the vibration amplitudes were completely attenuated for all controllers in less than $1 \mathrm{~s}$. The robustness of the controllers is demonstrated by the envelope size (blue lines). The shortest envelope was observed in the Neuro-Fuzzy control, while the largest in the LQR/LMI control. The very same tendency was observed in the control effort, in accordance with Figures 10 (a) and 10(b).

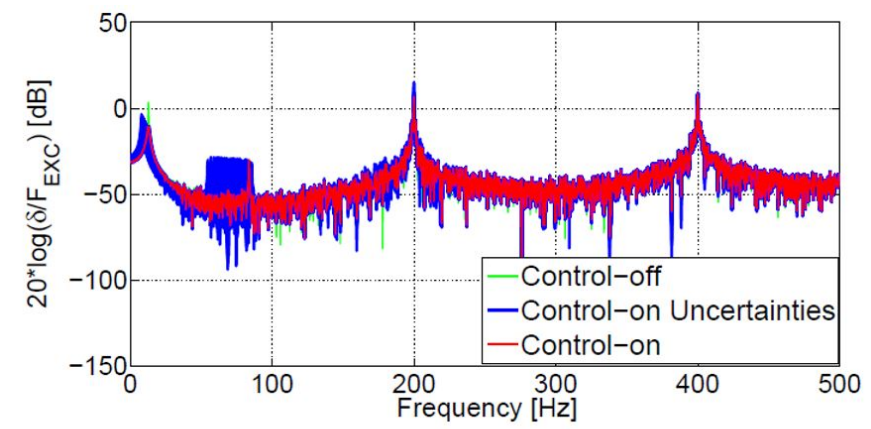

(a) LQR/LMI controller (case a)

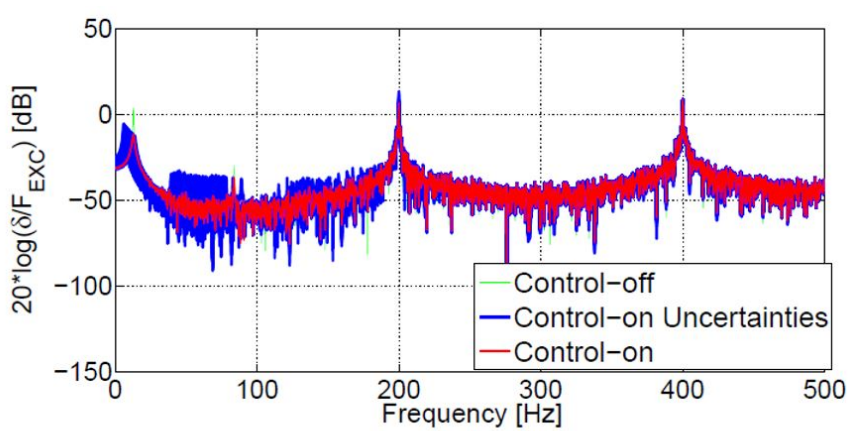

(b) Neuro-Fuzzy controller (case a)

Figure 9. Frequency response function LQR/LMI, and Neuro-fuzzy controllers for case (a).

Based on the FRFs (Figures 9(a) and 9(b)), the LQR/LMI controller presented a slightly shorter envelope than that from the other controller. However, only the Neuro-Fuzzy control attenuated the second mode.

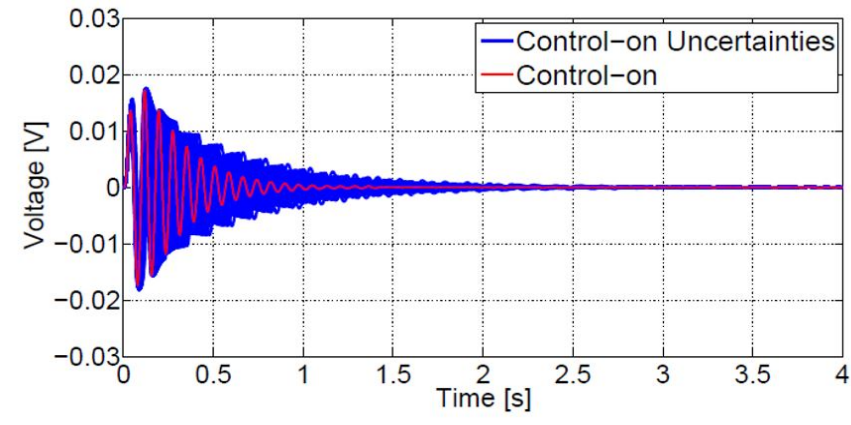

(a) LQR/LMI controller (case a)

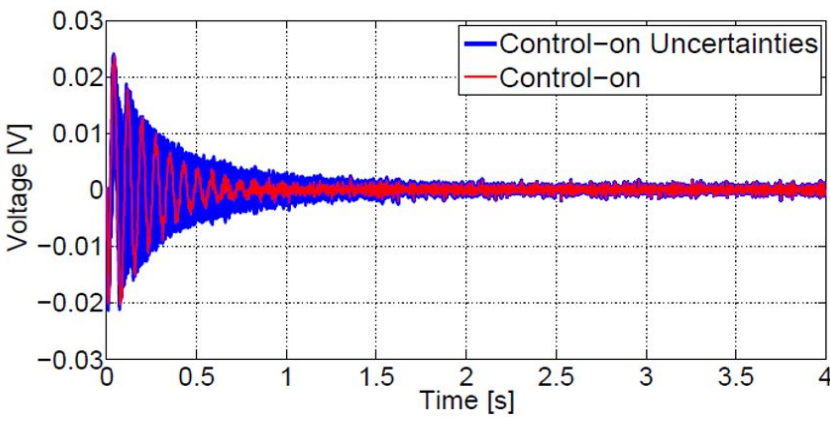

(b) Neuro-Fuzzy controller (case a)

Figure 10. Control effort of the LQR/LMI, and Neuro-fuzzy controllers for case (a).

The Neuro-Fuzzy control obtained a more robust result towards vibration attenuation considering the uncertainties in the dynamic matrix of the structure (case a). On the other hand, this controller demonstrated higher energy consumption due to the results of the control effort.

The deterministic results of the LQR/LMI and Neuro-Fuzzy controllers are shown in Figures 8 to 10 by the red lines.

By comparing the deterministic results of the impact response between the controllers and the system that was not controlled (Figures $8(a)$ and $8(b)$ ), the vibration was completely attenuated for all controllers, being higher with the Neuro-Fuzzy. However, this controller presented a higher necessary control effort (Figure 10 (a) and 10(b)).

Figures 9(a) and 9(b) illustrate the frequency response function (FRF). The first mode was attenuated for all controllers ( $14.10 \mathrm{~dB}$ for the LQR/LMI controller and $15.39 \mathrm{~dB}$ for the Neuro-Fuzzy controller). In the Neuro-Fuzzy control, there was an attenuation of $6.90 \mathrm{~dB}$ in the second mode, as shown in Figure 9(b). The results contemplating the uncertainties in the dynamic matrix of the Kalman estimator, that is, case (b), are depicted in Figures 11 to 13 . These results were divided into two groups, where the first comprises the results from the LQR/LMI controller (Figures 11(a), $12(a)$ and 13(a)), the second comprises the results from the Neuro-Fuzzy controller (Figures 11(b), 12(b) and 13(b)). 


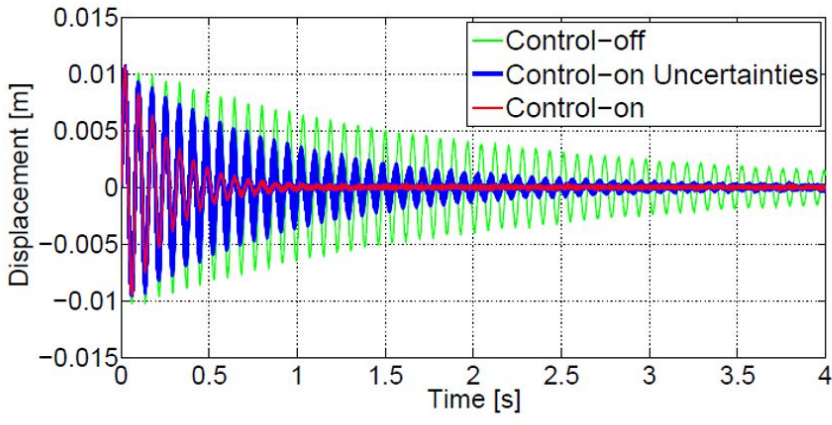

(a) LQR/LMI controller (case b)

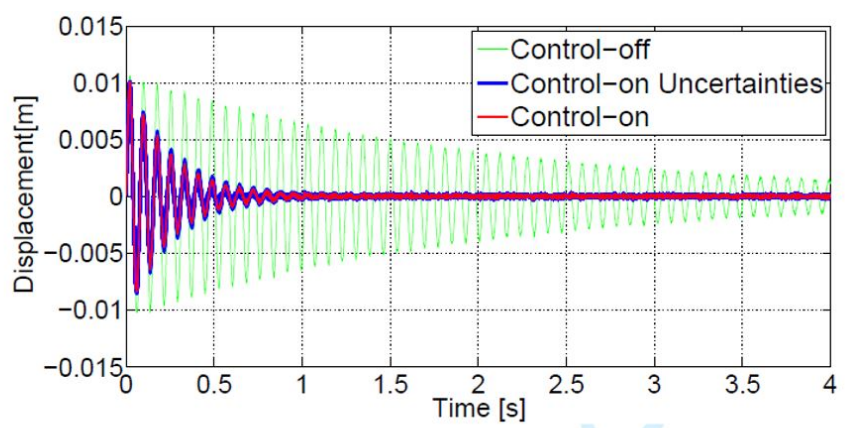

(b) Neuro-Fuzzy controller (case b)

Figure 11. Impact response of the LQR/LMI, and Neuro-fuzzy controllers for case (b).

Case (b) presented slightly shorter envelopes when compared to case (a), which might be attributed to the fact that the variation is applied to the dynamic matrix of the estimator. Whenever the variation is considered in the structure model, the natural frequency changes according to this variation, since the dynamic matrix also changes with $\Delta$ values.

Since the influence of variation applied to the dynamic matrix of the estimator is relatively smaller, the envelopes observed from the results of case (b) were shorter. Figure 11(b) suggests that the use of the Neuro-Fuzzy controller presented better responses and robustness in comparison to the other controllers. However, the control effort envelope for this controller increased during the first oscillations (Figure 13(b)).

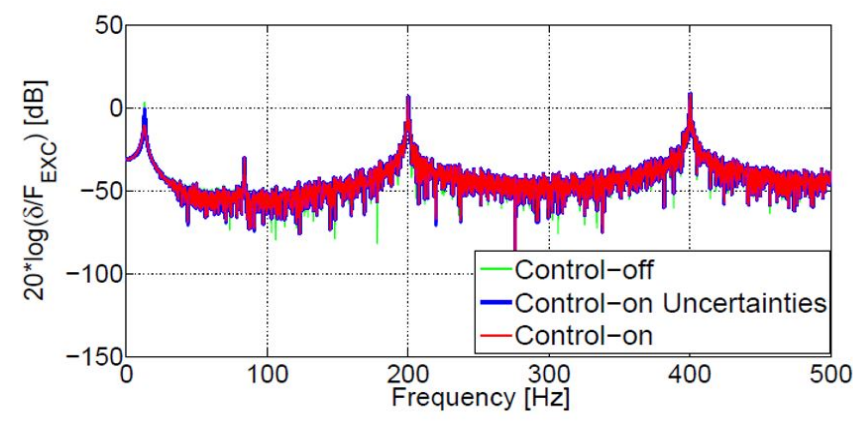

(a) LQR/LMI controller (case b)

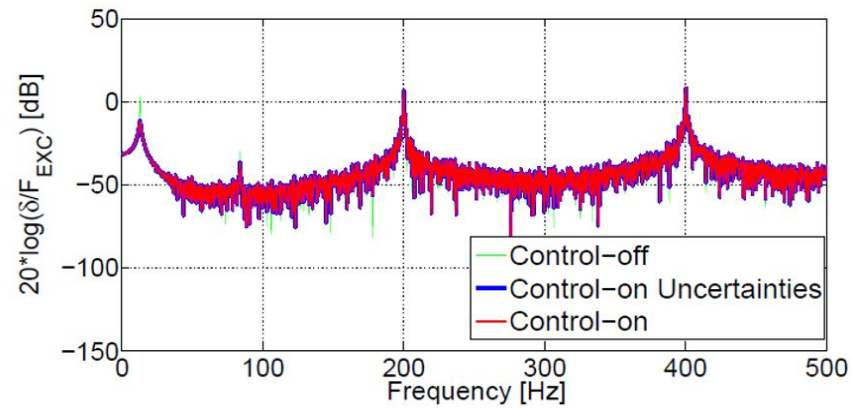

(b) Neuro-Fuzzy controller (case b)

Figure 12. Frequency response function of the LQR/LMI, and Neuro-fuzzy controllers for case (b).

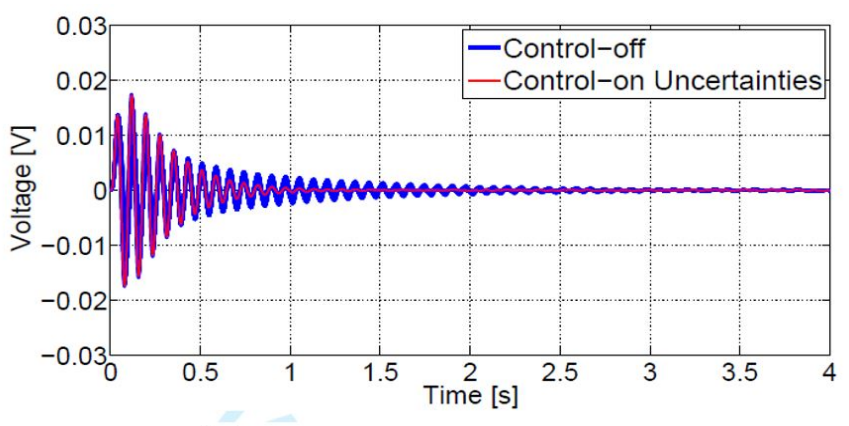

(a) LQR/LMI controller (case b)

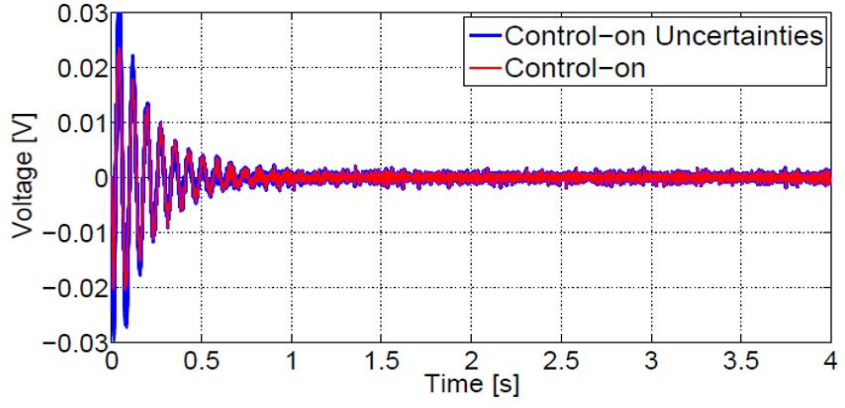

(b) Neuro-Fuzzy controller (case b)

Figure 13. Control effort of the LQR/LMI, and Neuro-fuzzy controllers for case (b).

The numerical results indicate that the Neuro-fuzzy controller exhibits better performance in terms of vibration attenuation compared with the LQR/LMI controller. Nevertheless, this improvement demands an increase in the control effort by the Neuro-Fuzzy controller. Therefore, there is a compromise between the control performance and control effort, i.e., the improvement in the vibration attenuation demands an increase in the control effort. The Neuro-Fuzzy controller has also presented a better performance against uncertainties.

This characteristic demonstrates that the Neuro-Fuzzy controller has a better performance to external disturbance and uncertainties when compared with linear robust control techniques. 


\section{CONCLUSIONS}

The effectiveness presented by the Layerwise-HSDT model in modelling thin and thick laminated composite plates which are free from transverse shear locking has been demonstrated. However, there are still controversial discussions regarding the application of mechanical boundary conditions to high-order theories in the scientific literature, which indicates a perceivable scenario for further research.

The increasing computing power of digital computers and the better approximation that has been verified in the HSDT models in relation to those incorporating FSDT formulations make HSDT a feasible and attractive theory to be combined with the Layerwise Theory to originate a mixed model. In this study, such mathematical modeling delineates the dynamic behavior of laminated composite structures with piezoelectric sensors and actuators. The so-called Mixed Theory allows the inclusion of piezoelectric materials in the surface of the laminated composite structure (inserts) or embedded inside.

Through a series of applications, this paper compared results from the Classical Laminate Theory (CLT), First-order Shear Deformation Theory (FSDT), High-order Shear Deformation Theory (HSDT) and the exact solution from the Theory of Elasticity with the Mixed Theory. Results show that the Mixed Theory is highly convenient to compute the dynamic (natural frequencies and vibration modes) and static (vertical displacement, electric potential difference) responses of thin and, particularly, thick laminated composite structures with piezoelectric elements.

Two specific applications described the modeling of a laminated composite plate with piezoelectric inserts attached to its surface (actuators) and a laminated composite beam with continuous piezoelectric layers (sensors) using the Mixed Theory. The numerical results, when compared with different HSDT models from the literature in terms of normalized vertical displacements and electric potential difference along the length of the structures, validated the electric-mechanical coupling of the proposed mixed model (formulated through Layerwise and HSDT theories). Thus, the Mixed Theory proved to be a reliable tool in the active control of thin and thick laminated composite structures with piezoelectric elements in both dynamic and static domains. Furthermore, it can be potentially useful in sensitivity analyzes, multi-objective robust optimization problems and several other numerical applications not investigated herein.

The analysis of active vibration control in the structure of a laminated composite material was made through two control techniques: LQR control solved by LMIs and Neuro-Fuzzy control. Reducing the model with the use of balanced realization was extremely important since this method ranks the modes according to their order of relevance concerning the system dynamic behavior. The vibration control analysis can be divided into two parts: deterministic control and robustness.

In the first part (deterministic case), it is possible to see the effectiveness presented for both controllers. In the deterministic control case, the system presents an equivalent steady response. The Frequency Response Functions results (FRFs) reveal that controlling the first mode would be enough to ensure satisfactory control, but by controlling the second mode a slight improvement in the vibration attenuation can be achieved with the neuro-fuzzy controller. Additionally, the spillover effect was not observed for superior modes.

The robustness analysis was split into two case studies. The first study consisted of applying uncertainties in the dynamical matrix of the model, which simulated modifications in the structure. In the second study, the uncertainties were applied in the dynamical matrix of the estimator that represents uncertainties in parameters of system identification. The comparison of responses from both controlled and non-controlled systems highlighted the valuable potential of the proposed control approach. Regarding cases (a) and (b), the Neuro-Fuzzy controller presented the most effective results in terms of vibration attenuation. Nevertheless, it required a higher control effort compared to the other controller. It is possible to see that the neuro-fuzzy controller was more robust than LQR solved by LMI. The proposed methodology was validated by numerical simulation, and the numerical results indicate that the neuro-fuzzy controller has benefits over the control approaches previously reported in the literature.

\section{Acknowledgements}

The authors gratefully acknowledge the financial support for this research from CNPq (Process 402581/2016-4 and 427204/2018-6), CAPES and Araucária Foundation.

Author's Contributions: Conceptualization, JP Repinaldo, AW Faria, R Alves e Silva, EH Koroishi and FA Lara-Molina; Methodology, JP Repinaldo, AW Faria and EH Koroishi; Investigation, JP Repinaldo, AW Faria, EH Koroishi and FA Lara-Molina; Writing - original draft, AW Faria, R Alves e Silva, EH Koroishi and FA Lara-Molina; Writing - review \& editing, AW Faria, 
R Alves e Silva, EH Koroishi and FA Lara-Molina; Funding acquisition, EH Koroishi and FA Lara-Molina; Resources, EH Koroishi and FA Lara-Molina.

Editor: Marco L. Bittencourt.

\section{References}

Anderson BDO and Moore JB (1979) Optimal Filtering, Prentice-Hall.

Assunção E, Marchesi HF, Teixeiria MCM and Peres PLD (2007) Global Optimization for the H1 - Norm Model Reduction Problem. International Journal of Systems Science (38): 125-138.

Chee CYK (2000) Static Shape Control of Laminated CompositePlate Smart Structure using Piezoelectric Actuators Ph.D.Thesis, University of Sydney, Sydney, Australia.

Conceição, S.M., Bueno, B.N., Cavalini Jr, A.A., Abreu, G.L., Melo, G.P. and Lopes Jr, V., 2009, "Model Reduction Methods for Smart Truss like Structure", Proceedings of 8th Brazilian Conference on Dynamics, Control and Applications, Bauru, Brasil.

Correia VMF, Gomes MAA, Suleman A, Soares CMM and Soares CAM (2000) Modeling and Design of Adaptive Composite Structures. Computer Methods in Applied Mechanics and Engineering 185: 325-346.

Crawley EF and Lazarus KB (1991) Induced Strain Actuation of Isotropic and Anisotropic Plates. AIAA Journal 29(6): 944-951.

Detwiler DT, Shen MHH and Venkayya VB (1995) Finite Element Analysis of Laminated Composite Structures Containing Distributed Piezoelectric Actuators and Sensors. Finite Elements in Analysis and Design 20(2): 87-100.

Durbin J, Koopman SJ (2002) Time Series Analysis by State Space Methods, Oxford University Press.

Elahi, H., Eugeni, M., \& Gaudenzi, P. (2018). A review on mechanisms for piezoelectric-based energy harvesters. Energies, 11(7), 1850.

Erkus B and Lee YJ (2004) Linear Matrix Inequalities and Matlab LMI Toolbox. Los Angeles, California: University of Southern California Group Meeting Report.

Faria AW (2006) Modelling of Composite Plates with Piezoelectric Sensors and Actuators via Finite Elements:Computational Implementation and Numerical Evaluation. Masters Thesis (in Portuguese). Federal University of Uberlândia - UFU, Uberlândia-MG, Brazil. Available at: https://repositorio.ufu.br/handle/123456789/15025 (accessed September 24, 2018).

Ganapathi M, Patel BP and Touratier MA (2004) C1 Finite Element for Flexural and Torsional Analysis of Rectangular Piezoelectric Laminated/Sandwich Composite Beams. International Journal for Numerical Methods in Engineering 61: 584-610.

Hwang, WS and Park, HC (1993) Finite Element Modeling of Piezoelectric Sensors and Actuators. AIAA Journal 31: 930-937.

Jang JSR (1993). ANFIS: Adaptive-Network-based Fuzzy Inference System. IEEE Transactions on Systems, Man, and Cybernetics 23(3).

Khare RK, Kant T and Garg AK (2003) Closed-Form Thermo-Mechanical Solutions of Higher-Order Theories of Cross-Ply Laminated Shallow Shells. Composite Structures 59(3): 313-340.

Koconis DB, Kollr LP and Springer GS (1994) Shape Control of Composite Plates and Shells with Embedded Actuators. I. Voltages Specified. Journal of Composite Materials 28(5): 415-458.

Koroishi E, Lara-Molina F, Faria AW and Steffen Jr, V. (2015) Robust Optimal Control Applied to a Composite Laminated Beam. Journal of Aerospace Technology and Management (Online) (7): 70-80.

Laub AJ, Heath MT, Paige CC andWard RC (1987) Computation of Systems Balancing Transformation and Other Applications of Simultaneous Diagonalization Algorithms. IEEE Transactions on Automatic Control 32: 115-122.

Lima Jr JJ (1999) Modelling of Piezoelectric Sensors and Actuators for Applications in the Active Control of Structures.PhD Thesis (in Portuguese). Campinas State University - Unicamp, Campinas-SP, Brazil. Available at: http://www.fem.unicamp.br/ dmchp/frame/portugues/laborato rios/vibrac/Juliano1999.pdf (accessed September 24, 2018).

Lin J and Zheng YB (2012) Vibration Suppression Control of Smart Piezoelectric Rotating Truss Structure by Parallel NeuroFuzzy Control with Genetic Algorithm Tuning. Journal of Sound and Vibration 331(16): 3677-3694. 
Manoharan R, Vasudevan R and Jeevanantham AK (2015) Vibration Analysis of a Partially Treated Laminated Composite Magnetorheological Fluid Sandwich Plate. Journal of Vibration and Control 22(3): 869-895.

Memmolo, V., Elahi, H., Eugeni, M., Monaco, E., Ricci, F., Pasquali, M., \& Gaudenzi, P. (2019). Experimental and numerical investigation of PZT response in composite structures with variable degradation levels. Journal of Materials Engineering and Performance, 28(6), 3239-3246.

Mirghaffari A and Rahmani B (2017) Active Vibration Control of Carbon Nanotube Reinforced Composite Beams. Transactions of the Institute of Measurement \& Control, 39(12): 1851-1863.

Mitchell R, Kim Y, El-Korchi T (2012) System Identification of Smart Structures using Wavelet Neuro-Fuzzy Model. Smart Materials and Structures. 21: 1-12.

Muradova ADM, Tairidis GK and Stavroulakis GE (2017) Adaptive Neuro-Fuzzy Vibration Control of a Smart Plate. Numerical Algebra. Control and Optimization 7(3): 251271.

Nasser H, Kiefer-Kamal EH, Hu H, Belouettar S and Barkanov (2012) Active Vibration Damping of Composite Structures using a Nonlinear Fuzzy Controller. Composite Structures 94(4): 1385-1390.

Ogata K (2010) Modern Control Engineering, 5th edn. Prentice Hall.

Rahmani B and Shenas AG (2017) Robust Vibration Control of Laminated Rectangular Composite Plates in Hygrothermal and Thermal Environment. Composite Structures, 179: 665-681.

Reddy JN (2003) Mechanics of Laminated Composite Plates andShells: Theory and Analysis, 2nd edn. CRC-Press.

Robbins DH, Reddy JN (1991) Analysis of Piezoelectrically Actuated Beams Using a Layer-wise Displacement Theory. Computers \& Structures 41(2): 265-279.

Saravanos DA, Heyliger PR (1995) Coupled Layerwise Analysis of Composite Beams with Embedded Piezoelectric Sensors.

Schulz SL, Gomes HM and Awruch AM (2013) Optimal Discrete Piezoelectric Patch Allocation on Composite Structures for Vibration Control Based on GA and Modal LQR. Computers \& Structures 128: 101-115.

Simões RC, Der Hagopian J, Mahfoud J and Steffen Jr V (2007) Modal Active Vibration Control of a Rotor using Piezoelectric Stack Actuators. Journal of Vibration and Control 13: 4564.

Thinh NT, Yang YS, and Oh IK (2009) Adaptive Neuro-Fuzzy Control of lonic Polymer Metal Composite Actuators. Smart Materials and Structures 18(6): 065016.

Tzou, H. S. and Ye, R. (1994). Piezothermoelasticity and Precision Control of Piezoelectric Systems: Theory and Finite Element Analysis. ASME. J. Vib. Acoust. October 1994; 116(4): 489-495. https://doi.org/10.1115/1.2930454

Tzou HS and Ye R (1996) Analysis of Piezoelectric Structures with Laminated Piezoelectric Triangle Shell Elements. AIAA Journal 34: 110-115.

Tzou HS, Tseng Cl (1990) Distributed Piezoelectric Sensor/ Actuator Designer for Dynamic Measurement/Control of Distributed Parameter Systems: a Piezoelectric Finite Element Approach. Journal of Sound and Vibration 138(1): 17-37.

Wang JZ, Wang XM and Zhou YH (2012) A Wavelet Approach for Active-Passive Vibration Control of Laminated Plates. Acta Mechanica Sinica 28(2): 520-531.

Welch G, Bishop G (1995) An Introduction to Kalman Filter Technical Report, University of North Carolina, Chapel Hill, USA.

Xu B, Ding H, Xie YM (2017) Optimal Design of Material Microstructure for Maximizing Damping Dissipation Velocity of Piezoelectric Composite Beam. International Journal of Mechanical Sciences, 128: 527-540.

Zhang S., Schmidt R and Xiansheng Q (2015) Active Vibration Control of Piezoelectric Bonded Smart Structures using PID Algorithm. Chinese Journal of Aeronautics 28(1): 305-313. 


\section{APPENDIX A}

The matrix $\left[A_{u}(z)\right]$ according to presently employed theory is expressed as follows:

$$
\left[A_{u}(z)\right]=\left[\begin{array}{ccccccccccc}
1 & 0 & 0 & z & 0 & 0 & z^{2} & 0 & 0 & z^{3} & 0 \\
0 & 1 & 0 & 0 & z & 0 & 0 & z^{2} & 0 & 0 & z^{3} \\
0 & 0 & 1 & 0 & 0 & z & 0 & 0 & z^{2} & 0 & 0
\end{array}\right]
$$

The matrix $[D(z)]$ incorporates flexural and transverse shear strains and assumes the following form:

$$
[D(z)]=\left[\begin{array}{ccccccccccc}
D_{x} & 0 & 0 & z D_{x} & 0 & 0 & z^{2} D_{x} & 0 & 0 & z^{3} D_{x} & 0 \\
0 & D_{y} & 0 & 0 & z D_{y} & 0 & 0 & z^{2} D_{y} & 0 & 0 & z^{3} D_{y} \\
0 & 0 & 0 & 0 & 0 & z & 0 & 0 & 2 z & 0 & 0 \\
0 & 0 & D_{y} & 0 & z & z D_{y} & 0 & 2 z & z^{2} D_{y} & 0 & 3 z^{2} \\
0 & 0 & D_{x} & z & 0 & z D_{x} & 2 z & 0 & z^{2} D_{x} & 3 z^{2} & 0 \\
D_{y} & D_{x} & 0 & z D_{y} & z D_{x} & 0 & z^{2} D_{y} & z^{2} D_{x} & 0 & z^{3} D_{y} & z^{3} D_{x}
\end{array}\right]
$$

Where: $D_{x}=\frac{\partial}{\partial_{x}}$ e $D_{y}=\frac{\partial}{\partial y}$ 